\title{
POLARIZED LINE FORMATION IN MULTI-DIMENSIONAL MEDIA.III. HANLE EFFECT WITH PARTIAL FREQUENCY REDISTRIBUTION
}

\author{
L. S. Anusha ${ }^{1}$ and K. N. Nagendra ${ }^{1}$ \\ ${ }^{1}$ Indian Institute of Astrophysics, Koramangala, 2nd Block, Bangalore 560 034, India
}

\begin{abstract}
In the previous two papers, namely, Anusha \& Nagendra (2011) and Anusha et al. (2011) we solved the polarized radiative transfer (RT) equation in multi-dimensional (multi-D) geometries, with partial frequency redistribution (PRD) as the scattering mechanism. We assumed Rayleigh scattering as the only source of linear polarization $(Q / I, U / I)$ in both these papers. In this paper we extend these previous works to include the effect of weak oriented magnetic fields (Hanle effect) on line scattering. We generalize the technique of Stokes vector decomposition in terms of the irreducible spherical tensors $\mathcal{T}_{Q}^{K}$, developed in Anusha \& Nagendra (2011), to the case of RT with Hanle effect. A fast iterative method of solution (based on the Stabilized Preconditioned Bi-Conjugate-Gradient technique), developed in Anusha et al. (2011), is now generalized to the case of RT in magnetized three-dimensional media. We use the efficient short-characteristics formal solution method for multi-D media, generalized appropriately to the present context. The main results of this paper are the following: (1) A comparison of emergent $(I, Q / I, U / I)$ profiles formed in one-dimensional (1D) media, with the corresponding emergent, spatially averaged profiles formed in multi-D media, shows that in the spatially resolved structures, the assumption of 1D may lead to large errors in linear polarization, especially in the line wings. (2) The multiD RT in semi-infinite non-magnetic media causes a strong spatial variation of the emergent $(Q / I, U / I)$ profiles, which is more pronounced in the line wings. (3) The presence of a weak magnetic field modifies the spatial variation of the emergent $(Q / I, U / I)$ profiles in the line core, by producing significant changes in their magnitudes.
\end{abstract}

Subject headings: line: formation - radiative transfer - polarization - scattering - magnetic fields - Sun: atmosphere

\section{INTRODUCTION}

Multi-dimensional (multi-D) radiative transfer (RT) is important to advance our understanding of the solar atmosphere. With the increase in the resolving power of modern telescopes, and the computing power of supercomputers, multi-D polarized line RT is becoming a necessity, and practically feasible. The multi-D effects manifest themselves in the resolved structures on the Sun. The finite dimensional structures on the solar surface lead to inhomogeneity in the atmosphere, which is then no longer axi-symmetric. The presence of magnetic fields adds to the non-axisymmetry, in the microscopic scales through the Hanle effect. The purpose of this paper is to address the relative importance of non-axisymmetry caused by geometry, and oriented magnetic fields.

In the past decades extensive studies on line RT in multi-D media are done. A historical account on these developments is given in Anusha \& Nagendra (2011, hereafter Paper I). In Paper I we presented a method of Stokes vector decomposition, which helped to formulate an 'irreducible form' of the polarized line transfer equation in a 3D Cartesian geometry. Such a formulation is advantageous because, the source vector and the mean intensity vector become angle independent in the reduced 
basis. Also the scattering phase matrix becomes independent of the outgoing directions $(\boldsymbol{\Omega})$. This property leads to several advantages in numerical work. It also provides a framework in which the transfer equation can be solved more conveniently, because the decomposition is applied to both the Stokes source vector, and the Stokes intensity vector. In Anusha et al. (2011, hereafter Paper II), we focused our attention on devising fast numerical methods to solve polarized RT equation with partial frequency redistribution (PRD) in a twodimensional (2D) geometry. In Paper I and Paper II we considered the case of non-magnetic resonance scattering polarization. Manso Sainz \& Trujillo Bueno (1999) and Dittmann (1999) solved the polarized RT equation in the presence of a magnetic field (Hanle effect), in multi-D media. Their calculations used the assumption of complete frequency redistribution (CRD) in line scattering. In this paper we solve the same problem, but for the more difficult and more realistic case of Hanle scattering with PRD. The physics of PRD scattering is treated using the frequency-domain based approach developed by Bommier (1997a,b). The RT calculations in one-dimensional (1D) geometry, using this approach are described in Nagendra et al. (2002). We extend their work to 2D and 3D geometries. For simplicity we restrict to the case of angle averaged PRD functions.

The present paper represents a generalization to the magnetic case, the decomposition technique developed in Paper I. It also represents the generalizations to the 3D case, the Stabilized Pre-conditioned Bi-Conjugate Gradient (preBiCG-STAB) method developed in Paper II. Another generalization is the use of $3 \mathrm{D}$ short characteristics formal solver in this paper, for the case of PRD.

In Section 2 we describe the multi-D transfer equation in the Stokes vector basis. The decomposition technique as applied to the case of a magnetic multi-D media is described in Section 3. In Section 4 we briefly describe the 3D short characteristics formal solution method. Section 5 is devoted to a brief description of the numerical method of solution. Results and discussions are presented in Section 6. Conclusions are given in Section 7 .

\section{THE POLARIZED HANLE SCAT- TERING LINE TRANSFER EQUA- TION IN MULTI-D MEDIA}

In this paper we consider polarized RT in 1D, 2D and 3D media in Cartesian geometry (see Figure 1). We assume that the $1 \mathrm{D}$ medium is infinite in the $X$ and $Y$ directions but finite in the $Z$ direction. For $2 \mathrm{D}$, we assume that the medium is infinite in the $X$ direction, but finite in the $Y$ and $Z$ directions. The 3D medium is assumed to be finite in all the $X, Y$ and $Z$ directions. We define the "top surface" for a $1 \mathrm{D}$ medium to be the infinite $X Y$ plane passing through the point $Z_{\max }$. For a $2 \mathrm{D}$ medium, the top surface is defined to be the plane passing through the line $\left(Y, Z_{\max }\right)$, which is infinite in $X$ direction. For a $3 \mathrm{D}$ medium, the top surface is the plane $\left(X, Y, Z_{\max }\right)$ which is finite in $X$ and $Y$ directions. For a given ray with direction $\boldsymbol{\Omega}$, the polarized transfer equation in a multi-D medium with an oriented magnetic field is given by

$$
\begin{aligned}
& \boldsymbol{\Omega} \cdot \boldsymbol{\nabla} \boldsymbol{I}(\boldsymbol{r}, \boldsymbol{\Omega}, x)=-\left[\kappa_{l}(\boldsymbol{r}) \phi(x)+\kappa_{c}(\boldsymbol{r})\right] \\
& \times[\boldsymbol{I}(\boldsymbol{r}, \boldsymbol{\Omega}, x)-\boldsymbol{S}(\boldsymbol{r}, \boldsymbol{\Omega}, x)],
\end{aligned}
$$

where $\boldsymbol{I}=(I, Q, U)^{T}$ is the Stokes vector, with $I, Q$ and $U$ the Stokes parameters defined below. Following Chandrasekhar (1960), we consider an elliptically polarized beam of light, the vibrations of the electric vector of which describe an ellipse. If $I_{l}$ and $I_{r}$ denote the components of the specific intensity of this beam of light along two mutually perpendicular directions $l$ and $r$, in a plane (see Figure 2) transverse to the propagation direction, then we define

$$
\begin{array}{r}
I=I_{l}+I_{r}, \\
Q=I_{l}-I_{r}, \\
U=\left(I_{l}-I_{r}\right) \tan 2 \chi,
\end{array}
$$

where $\chi$ is the angle between the direction $l$ and the semi-major axis of the ellipse. Positive value of $Q$ is defined to be a direction parallel to $l$ and negative $Q$ to be in a direction parallel to $r$. The quantity $\boldsymbol{r}=(\mathrm{x}, \mathrm{y}, \mathrm{z})$ is the position vector of the ray in the Cartesian co-ordinate system. The unit vector $\boldsymbol{\Omega}=(\eta, \gamma, \mu)=(\sin \theta \cos \varphi, \sin \theta \sin \varphi, \cos \theta)$ describes the direction cosines of the ray in the atmosphere, with respect to the atmospheric normal (the $Z$-axis), where $\theta$ and $\varphi$ are the polar and 
azimuthal angles of the ray (see Figure 2). The quantity $\kappa_{l}$ is the frequency averaged line opacity, $\phi$ is the Voigt profile function and $\kappa_{c}$ is the continuum opacity. Frequency is measured in reduced units, namely $x=\left(\nu-\nu_{0}\right) / \Delta \nu_{D}$, where $\Delta \nu_{D}$ is the Doppler width. The Stokes source vector in a two-level atom model with unpolarized ground level is

$$
\begin{aligned}
& \boldsymbol{S}(\boldsymbol{r}, \boldsymbol{\Omega}, x) \\
& =\frac{\kappa_{l}(\boldsymbol{r}) \phi(x) \boldsymbol{S}_{l}(\boldsymbol{r}, \boldsymbol{\Omega}, x)+\kappa_{c}(\boldsymbol{r}) \boldsymbol{S}_{c}(\boldsymbol{r}, x)}{\kappa_{l}(\boldsymbol{r}) \phi(x)+\kappa_{c}(\boldsymbol{r})} .
\end{aligned}
$$

Here $\boldsymbol{S}_{c}$ is the continuum source vector given by $\left(B_{\nu}(\boldsymbol{r}), 0,0\right)^{T}$ with $B_{\nu}(\boldsymbol{r})$ being the Planck function. The line source vector is written as

$$
\begin{aligned}
& \boldsymbol{S}_{l}(\boldsymbol{r}, \boldsymbol{\Omega}, x)=\boldsymbol{G}(\boldsymbol{r})+\int_{-\infty}^{+\infty} d x^{\prime} \\
& \times \oint \frac{d \boldsymbol{\Omega}^{\prime}}{4 \pi} \frac{\hat{R}\left(x, x^{\prime}, \boldsymbol{\Omega}, \boldsymbol{\Omega}^{\prime}, \boldsymbol{B}\right)}{\phi(x)} \boldsymbol{I}\left(\boldsymbol{r}, \boldsymbol{\Omega}^{\prime}, x^{\prime}\right) .
\end{aligned}
$$

Here $\hat{R}$ is the Hanle redistribution matrix and $\boldsymbol{B}$ represents an oriented vector magnetic field. $\epsilon=\Gamma_{I} /\left(\Gamma_{R}+\Gamma_{I}\right)$ with $\Gamma_{I}$ and $\Gamma_{R}$ being the inelastic collision rate and the radiative de-excitation rate respectively. The thermalization parameter $\epsilon$ is the rate of photon destruction by inelastic collisions. The damping parameter is computed using $a=a_{R}\left[1+\left(\Gamma_{E}+\Gamma_{I}\right) / \Gamma_{R}\right]$ where $a_{R}=\Gamma_{R} / 4 \pi \Delta \nu_{D}$ and $\Gamma_{E}$ is the elastic collision rate. We denote the thermal source vector by $\boldsymbol{G}(\boldsymbol{r})=\epsilon \boldsymbol{B}_{\nu}(\boldsymbol{r})$ with $\boldsymbol{B}_{\nu}(\boldsymbol{r})=\left(B_{\nu}(\boldsymbol{r}), 0,0\right)^{T}$. The solid angle element $d \boldsymbol{\Omega}^{\prime}=\sin \theta^{\prime} d \theta^{\prime} d \varphi^{\prime}$, where $\theta \in[0, \pi]$ and $\varphi \in[0,2 \pi]$. The transfer equation along the ray path takes the form

$$
\begin{aligned}
& \frac{d \boldsymbol{I}(\boldsymbol{r}, \boldsymbol{\Omega}, x)}{d s} \\
& =-\kappa_{\text {tot }}(\boldsymbol{r}, x)[\boldsymbol{I}(\boldsymbol{r}, \boldsymbol{\Omega}, x)-\boldsymbol{S}(\boldsymbol{r}, \boldsymbol{\Omega}, x)] .
\end{aligned}
$$

The formal solution of Equation (5) is given by

$$
\begin{aligned}
& \boldsymbol{I}(\boldsymbol{r}, \boldsymbol{\Omega}, x) \\
& =\boldsymbol{I}\left(\boldsymbol{r}_{0}, \boldsymbol{\Omega}, x\right) \exp \left\{-\int_{s_{0}}^{s} \kappa_{\mathrm{tot}}\left(\boldsymbol{r}-s^{\prime \prime} \boldsymbol{\Omega}, x\right) d s^{\prime \prime}\right\} \\
& \quad+\int_{s_{0}}^{s} \boldsymbol{S}\left(\boldsymbol{r}-s^{\prime} \boldsymbol{\Omega}, \boldsymbol{\Omega}, x\right) \kappa_{\mathrm{tot}}\left(\boldsymbol{r}-s^{\prime} \boldsymbol{\Omega}, x\right) \\
& \quad \times \exp \left\{-\int_{s^{\prime}}^{s} \kappa_{\mathrm{tot}}\left(\boldsymbol{r}-s^{\prime \prime} \boldsymbol{\Omega}, x\right) d s^{\prime \prime}\right\} d s^{\prime} .
\end{aligned}
$$

$\boldsymbol{I}\left(\boldsymbol{r}_{0}, \boldsymbol{\Omega}, x\right)$ is the boundary condition imposed at $\boldsymbol{r}_{0}=\left(\mathrm{x}_{0}, \mathrm{y}_{0}, \mathrm{z}_{0}\right)$. The ray path on which the formal solution is defined is shown in Figure 3.

\section{Decomposition of $S$ and $I$ for multi-D transfer in the presence of a magnetic field}

As already discussed in Paper I, a decomposition of the Stokes source vector $\boldsymbol{S}$ and the intensity vector $\boldsymbol{I}$ in terms of the irreducible spherical tensors is necessary to simplify the problem. In Paper I, it was a generalization to the 3D nonmagnetic case, of the decomposition technique for the 1D transfer problems, developed by Frisch (2007, hereafter HF07). Here we extend our work of Paper I to include the magnetic fields. A similar technique, but in the Fourier space was presented in Faurobert-Scholl (1991) and Nagendra et al. (1998), who solved the Hanle scattering RT problem in 1D geometry. The solution of polarized Hanle scattering transfer equation using the angle averaged and angle dependent redistribution matrices was presented in Nagendra et al. (2002), where a perturbation method of solution was used. A Polarized Approximate Lambda Iteration method to solve similar problems, using the Fourier decomposition technique was presented in Fluri et al. (2003), but only for the case of angle averaged PRD.

A general theory of PRD for the 2-level atom problem with Hanle scattering was developed by Bommier (1997a,b). It involves the construction of PRD matrices that describe radiative plus collisional frequency redistribution in scattering. It is rather difficult to use the exact redistribution matrix $\hat{R}$ in the polarized transfer equation. For convenience of applications in line transfer theories, Bommier (1997b) proposed 3 levels of approximations, to handle the $\hat{R}$ matrices. In approxima- 
tion levels 2 and 3 , the $\hat{R}$ matrices were factorized into products of redistribution functions of Hummer (1962), and the multi-polar components of the Hanle phase matrix. The collisions enter naturally in this formalism. It is shown that such a factorization of $\hat{R}$ can be achieved only in certain frequency domains in the 2-dimensional $\left(x, x^{\prime}\right)$ frequency space. In this paper we refer to this way of writing the PRD Hanle $\hat{R}$ matrix, as the 'domain based PRD'. The definition of the domains are given in Bommier (1997b) (see also Nagendra et al. 2002, 2003; Fluri et al. 2003). We use the domain based PRD, but write the relevant equations in a form suitable for our present context (multiD transfer). We recall that in the special case of non-magnetic scattering, the domain based PRD equations for $\hat{R}$ matrix naturally go to the DomkeHubeny redistribution matrix (Domke \& Hubeny 1988). We start by writing Hanle phase matrix in the atmospheric reference frame in terms of the irreducible spherical tensors for polarimetry, introduced by Landi Degl'Innocenti \& Landolfi (2004, hereafter LL04). In this formalism the $(i, j)$-th element of the Hanle phase matrix is given by

$$
\begin{aligned}
& {\left[\hat{P}_{H}\left(\boldsymbol{\Omega}, \boldsymbol{\Omega}^{\prime}, \boldsymbol{B}\right)\right]_{i j}=} \\
& \sum_{K Q} \mathcal{T}_{Q}^{K}(i, \boldsymbol{\Omega}) \sum_{Q^{\prime}} \mathcal{M}_{Q Q^{\prime}}^{K}(\boldsymbol{B})(-1)^{Q^{\prime}} \mathcal{T}_{-Q^{\prime}}^{K}\left(j, \boldsymbol{\Omega}^{\prime}\right),
\end{aligned}
$$

where $(i, j)=(1,2,3)$ and

$$
\begin{aligned}
& \mathcal{M}_{Q Q^{\prime}}^{K}(\boldsymbol{B})= \\
& e^{i\left(Q^{\prime}-Q\right) \chi_{B}} \sum_{Q^{\prime \prime}} d_{Q Q^{\prime \prime}}^{K}\left(\theta_{B}\right) d_{Q^{\prime \prime} Q^{\prime}}^{K}\left(-\theta_{B}\right) \frac{1}{1+i Q^{\prime \prime} \Gamma_{B}},
\end{aligned}
$$

where the $d_{M M^{\prime}}^{J}$ are the reduced rotation matrices given in LL04. The magnetic Hanle $\Gamma_{B}$ parameter takes different values in different frequency domains (see Appendix B). $\mathcal{T}_{Q}^{K}(i, \boldsymbol{\Omega})$ are the irreducible spherical tensors for polarimetry with $K=0,1,2,-K \leq Q \leq+K$ (see Landi Degl'Innocenti \& Landolfi 2004). In this paper, we consider only the linear polarization. Therefore, $K=0,2$ and $Q \in[-K,+K]$. For the practical use, we need to further expand the $\hat{P}_{H}$ matrix in each of the domains in terms of $\mathcal{T}_{Q}^{K}$. The required domain based expansions of the PRD matrices in terms of $\mathcal{T}_{Q}^{K}$ were already given in HF07, applicable there to the case of 1D Hanle transfer. We present here the corresponding equations that are applicable to the multi-D transfer, which now become $\varphi$ dependent (in the 1D case, those phase matrix components were $\varphi$ independent). We restrict our attention in this paper to the particular case of angle averaged redistribution functions (approximation level 3 of Bommier 1997b).

The $i j$-th element of the redistribution matrix in the atmospheric reference frame (Bommier 1997b) can be written as

$$
\begin{gathered}
R_{i j}\left(x, x^{\prime}, \boldsymbol{\Omega}, \boldsymbol{\Omega}^{\prime}, \boldsymbol{B}\right)=\sum_{K Q} W_{K} \mathcal{T}_{Q}^{K}(i, \boldsymbol{\Omega}) \\
\times\left\{r_{\mathrm{II}}\left(x, x^{\prime}\right) P_{Q, \mathrm{II}}^{K}\left(j, \boldsymbol{\Omega}^{\prime}, \boldsymbol{B}\right)\right. \\
\left.\quad+r_{\mathrm{III}}\left(x, x^{\prime}\right) P_{Q, \mathrm{III}}^{K}\left(j, \boldsymbol{\Omega}^{\prime}, \boldsymbol{B}\right)\right\} .
\end{gathered}
$$

The weights $W_{K}$ depend on the line under consideration (see LL04). Here $r_{\mathrm{II}}\left(x, x^{\prime}\right)$ and $r_{\mathrm{III}}\left(x, x^{\prime}\right)$ are the angle-averaged versions of redistribution functions (see Hummer 1962). The quantities $P_{Q, \mathrm{II}}^{K}\left(j, \boldsymbol{\Omega}^{\prime}, \boldsymbol{B}\right)$ and $P_{Q, \mathrm{III}}^{K}\left(j, \boldsymbol{\Omega}^{\prime}, \boldsymbol{B}\right)$ take different forms in different frequency domains. They are described in Appendix B.

Denoting $G_{Q}^{K}=\delta_{K 0} \delta_{Q 0} G(\boldsymbol{r})$, where $G(\boldsymbol{r})=$ $\epsilon B_{\nu}(\boldsymbol{r})$, we can write the $i$-th component of the thermal source vector as

$$
G_{i}(\boldsymbol{r})=\sum_{K Q} \mathcal{T}_{Q}^{K}(i, \boldsymbol{\Omega}) G_{Q}^{K}(\boldsymbol{r})
$$

The line source vector can be decomposed as

$$
S_{i, l}(\boldsymbol{r}, \boldsymbol{\Omega}, x)=\sum_{K Q} \mathcal{T}_{Q}^{K}(i, \boldsymbol{\Omega}) S_{Q, l}^{K}(\boldsymbol{r}, x),
$$

where

$$
\begin{aligned}
& S_{Q, l}^{K}(\boldsymbol{r}, x)=G_{Q}^{K}(\boldsymbol{r})+\frac{1}{\phi(x)} \int_{-\infty}^{+\infty} d x^{\prime} \oint \frac{d \boldsymbol{\Omega}^{\prime}}{4 \pi} \\
& \times \sum_{j=0}^{3} W_{K}\left\{r_{\mathrm{II}}\left(x, x^{\prime}\right) P_{Q, \mathrm{II}}^{K}\left(j, \boldsymbol{\Omega}^{\prime}, \boldsymbol{B}\right)\right. \\
& \left.\quad+r_{\mathrm{III}}\left(x, x^{\prime}\right) P_{Q, \mathrm{III}}^{K}\left(j, \boldsymbol{\Omega}^{\prime}, \boldsymbol{B}\right)\right\} I_{j}\left(\boldsymbol{r}, \boldsymbol{\Omega}^{\prime}, x^{\prime}\right) .
\end{aligned}
$$

Note that the components $S_{Q, l}^{K}(\boldsymbol{r}, x)$ now depend only on the spatial variables $(\mathrm{x}, \mathrm{y}, \mathrm{z})$, frequency $x$. The $(\theta, \varphi)$ dependence is fully contained in 
$\mathcal{T}_{Q}^{K}(i, \boldsymbol{\Omega})$. These quantities are listed in LL04 (chapter 5, Table 5.6, p. 211). Substituting Equation (11) in Equation (6), the components of $\boldsymbol{I}$ can be written as

$$
I_{i}(\boldsymbol{r}, \boldsymbol{\Omega}, x)=\sum_{K Q} \mathcal{T}_{Q}^{K}(i, \boldsymbol{\Omega}) I_{Q}^{K}(\boldsymbol{r}, \boldsymbol{\Omega}, x),
$$

where

$$
\begin{aligned}
& I_{Q}^{K}(\boldsymbol{r}, \boldsymbol{\Omega}, x)=I_{Q, 0}^{K}\left(\boldsymbol{r}_{0}, \boldsymbol{\Omega}, x\right) e^{-\tau_{x, \max }} \\
& +\int_{0}^{\tau_{x, \max }} e^{-\tau_{x}^{\prime}\left(\boldsymbol{r}^{\prime}\right)}\left[p_{x} S_{Q, l}^{K}\left(\boldsymbol{r}^{\prime}, x\right)\right. \\
& \left.+\left(1-p_{x}\right) S_{Q, C}^{K}\left(\boldsymbol{r}^{\prime}, x\right)\right] d \tau_{x}^{\prime}\left(\boldsymbol{r}^{\prime}\right) .
\end{aligned}
$$

Here $I_{Q, 0}^{K}=I_{0}\left(\boldsymbol{r}_{0}, \boldsymbol{\Omega}, x\right) \delta_{K 0} \delta_{Q 0}$ are the intensity components at the lower boundary. The quantities $S_{Q, C}^{K}=S_{C}(\boldsymbol{r}, x) \delta_{K 0} \delta_{Q 0}$ denote the continuum source vector components. We assume that $S_{C}(\boldsymbol{r}, x)=B_{\nu}(\boldsymbol{r})$. The ratio of the line opacity to the total opacity is given by

$$
p_{x}=\kappa_{l}(\boldsymbol{r}) \phi(x) / \kappa_{\mathrm{tot}}(\boldsymbol{r}, x) .
$$

The monochromatic optical depth scale is defined as

$$
\tau_{x}(\mathrm{x}, \mathrm{y}, \mathrm{z})=\int_{s_{0}}^{s} \kappa_{\mathrm{tot}}\left(\boldsymbol{r}-s^{\prime \prime} \boldsymbol{\Omega}, x\right) d s^{\prime \prime},
$$

where $\tau_{x}$ is measured along a given ray determined by the direction $\boldsymbol{\Omega}$. In Equation (14) $\tau_{x \text {,max }}$ is the maximum monochromatic optical depth at frequency $x$, when measured along the ray.

\subsection{The irreducible transfer equation in multi-D geometry for the Hanle scat- tering problem}

Let $S_{Q}^{K}=p_{x} S_{Q, l}^{K}+\left(1-p_{x}\right) S_{Q, C}^{K} \cdot I_{Q}^{K}$ and $S_{Q}^{K}$ as well as the phase matrix elements $P_{Q, \mathrm{II}}^{K}\left(j, \boldsymbol{\Omega}^{\prime}, \boldsymbol{B}\right)$ and $P_{Q, \mathrm{III}}^{K}\left(j, \boldsymbol{\Omega}^{\prime}, \boldsymbol{B}\right)$ are all complex quantities. Following the method of transformation from complex to the real quantities given in HF07, we define the real irreducible Stokes vector $\mathcal{I}=\left(I_{0}^{0}, I_{0}^{2}, I_{1}^{2, \mathrm{x}}\right.$, $\left.I_{1}^{2, \mathrm{y}}, I_{2}^{2, \mathrm{x}}, I_{2}^{2, \mathrm{y}}\right)^{T}$ and the real irreducible source vector $\mathcal{S}=\left(S_{0}^{0}, S_{0}^{2}, S_{1}^{2, \mathrm{x}}, S_{1}^{2, \mathrm{y}}, S_{2}^{2, \mathrm{x}}, S_{2}^{2, \mathrm{y}}\right)^{T}$. It can be shown that the $\mathcal{I}$ and $\mathcal{S}$ satisfy a transfer equation of the form

$$
\begin{aligned}
& -\frac{1}{\kappa_{\text {tot }}(\boldsymbol{r}, x)} \boldsymbol{\Omega} \cdot \boldsymbol{\nabla} \mathcal{I}(\boldsymbol{r}, \boldsymbol{\Omega}, x)= \\
& {[\mathcal{I}(\boldsymbol{r}, \boldsymbol{\Omega}, x)-\mathcal{S}(\boldsymbol{r}, x)],}
\end{aligned}
$$

where $\mathcal{S}(\boldsymbol{r}, x)=p_{x} \mathcal{S}_{l}(\boldsymbol{r}, x)+\left(1-p_{x}\right) \mathcal{S}_{C}(\boldsymbol{r}, x)$ with

$$
\begin{aligned}
& \mathcal{S}_{l}(\boldsymbol{r}, x)=\epsilon \boldsymbol{B}_{\nu}(\boldsymbol{r}) \\
& +\frac{1}{\phi(x)} \int_{-\infty}^{+\infty} d x^{\prime} \oint \frac{d \boldsymbol{\Omega}^{\prime}}{4 \pi} \hat{W}\left\{\hat{M}_{\mathrm{II}}^{(i)}(\boldsymbol{B}) r_{\mathrm{II}}\left(x, x^{\prime}\right)\right. \\
& \left.+\hat{M}_{\mathrm{III}}^{(i)}(\boldsymbol{B}) r_{\mathrm{III}}\left(x, x^{\prime}\right)\right\} \hat{\Psi}\left(\boldsymbol{\Omega}^{\prime}\right) \mathcal{I}\left(\boldsymbol{r}, \boldsymbol{\Omega}^{\prime}, x^{\prime}\right),
\end{aligned}
$$

and $\boldsymbol{S}_{C}(\boldsymbol{r}, x)=\left(S_{C}(\boldsymbol{r}, x), 0,0,0,0,0\right)^{T} . \hat{W}$ is a diagonal matrix given by

$$
\hat{W}=\operatorname{diag}\left\{W_{0}, W_{2}, W_{2}, W_{2}, W_{2}, W_{2}\right\} .
$$

The matrix $\hat{\Psi}$ represents the phase matrix for the Rayleigh scattering, to be used in multi-D geometries. Its elements are listed in Appendix D. The matrices $\hat{M}_{\mathrm{II}, \mathrm{III}}^{(i)}(\boldsymbol{B})$ in different domains are given in Appendix C. The formal solution now takes the form

$$
\begin{aligned}
& \mathcal{I}(\boldsymbol{r}, \boldsymbol{\Omega}, x)=\mathcal{I}\left(\boldsymbol{r}_{0}, \boldsymbol{\Omega}, x\right) e^{-\tau_{x, \max }} \\
& +\int_{0}^{\tau_{x, \max }} e^{-\tau_{x}^{\prime}\left(\boldsymbol{r}^{\prime}\right)} \mathcal{S}\left(\boldsymbol{r}^{\prime}, x\right) d \tau_{x}^{\prime}\left(\boldsymbol{r}^{\prime}\right)
\end{aligned}
$$

Here $\mathcal{I}\left(\boldsymbol{r}_{0}, \boldsymbol{\Omega}, x\right)$ is the boundary condition imposed at $\boldsymbol{r}_{0}$.

\section{A 3D FORMAL SOLVER BASED ON THE SHORT CHARACTERISTICS APPROACH}

This section is devoted to a discussion of $3 \mathrm{D}$ short characteristics formal solver. Here we generalize to the $3 \mathrm{D}$ case, the $2 \mathrm{D}$ short characteristics formal solver that we had used in Paper II. A short characteristic stencil MOP of a ray passing through the point $\mathrm{O}$, in a $3 \mathrm{D}$ cube is shown in Figure 4. The point $\mathrm{O}$ represents a grid point along the ray path. The point $\mathrm{M}$ (or P) represents an intersection of the ray with one of the boundary planes of a 3D cell. The plane of intersection is determined by the direction cosines of the ray. 
The length $\Delta s$ of the line segment MO (or OP) is given by

$$
\begin{array}{ll}
\Delta s=\Delta \mathrm{z} / \mu, & \text { if the ray hits the } X Y \text { plane, } \\
\Delta s=\Delta \mathrm{y} / \gamma, & \text { if the ray hits the } X Z \text { plane, } \\
\Delta s=\Delta \mathrm{x} / \eta, & \text { if the ray hits the } Y Z \text { plane. }
\end{array}
$$

Here $\Delta \mathrm{x}, \Delta \mathrm{y}$ and $\Delta \mathrm{z}$ are incremental lengths (positive or negative) between two successive grid points on the $X, Y$ and $Z$ directions respectively. In the short characteristics method, the irreducible Stokes vector $\mathcal{I}$ at $\mathrm{O}$ is given by

$$
\begin{aligned}
& \mathcal{I}_{\mathrm{O}}(\boldsymbol{r}, \boldsymbol{\Omega}, x)=\mathcal{I}_{\mathrm{M}}(\boldsymbol{r}, \boldsymbol{\Omega}, x) \exp \left[-\Delta \tau_{\mathrm{M}}\right] \\
& +\psi_{\mathrm{M}}(\boldsymbol{r}, \boldsymbol{\Omega}, x) \mathcal{S}_{\mathrm{M}}(\boldsymbol{r}, x) \\
& +\psi_{\mathrm{O}}(\boldsymbol{r}, \boldsymbol{\Omega}, x) \mathcal{S}_{\mathrm{O}}(\boldsymbol{r}, x) \\
& +\psi_{\mathrm{P}}(\boldsymbol{r}, \boldsymbol{\Omega}, x) \mathcal{S}_{\mathrm{P}}(\boldsymbol{r}, x)
\end{aligned}
$$

where $\mathcal{S}_{\mathrm{M}, \mathrm{O}, \mathrm{P}}$ are the irreducible source vectors at $\mathrm{M}, \mathrm{O}$ and $\mathrm{P}$. The quantity $\boldsymbol{\mathcal { I }}_{\mathrm{M}}$ is the upwind irreducible Stokes vector for the point O. If $\mathrm{M}$ and $\mathrm{P}$ are non-grid points, then $\mathcal{S}_{\mathrm{M}, \mathrm{P}}$ and $\mathcal{I}_{\mathrm{M}}$ are computed using a two-dimensional parabolic interpolation formula. While computing them, one has to ensure the monotonicity of all the 6 components of these vectors, through appropriate logical tests (see Auer \& Paletou 1994). The coefficients $\psi$ depend on the optical depth increments in $X$, $Y$ and $Z$ directions. For a 2D geometry, these coefficients are given in Auer \& Paletou (1994). Here we have used a generalized version of these coefficients, that are applicable to a 3D geometry.

\section{NUMERICAL METHOD OF SOLU- TION}

In this paper we generalize the pre-BiCG-STAB method described in Paper II to the case of a 3D geometry. The present work represents also an extension of this technique to the case of polarized $\mathrm{RT}$ in the presence of an oriented magnetic field. The essential difference between the 2D and 3D algorithms is in terms of the lengths of the vectors. In a $2 \mathrm{D}$ geometry it is $n_{p} \times n_{x} \times n_{Y} \times n_{Z}$ whereas in a 3D geometry it is $n_{p} \times n_{x} \times n_{X} \times n_{Y} \times n_{Z}$, where $n_{X, Y, Z}$ are the number of grid points in the $X, Y$ and $Z$ directions, and $n_{x}$ refers to the number of frequency points. $n_{p}$ is the number of polarization components of the irreducible vectors. In the presence of a magnetic field, $n_{p}=6$ in both $2 \mathrm{D}$ and $3 \mathrm{D}$ geometries. In non-magnetic problems, $n_{p}=4,6$ for $2 \mathrm{D}$ and $3 \mathrm{D}$ geometries respectively.

\subsection{The Preconditioner matrix}

A description of the preconditioner matrix that appears in the pre-BiCG-STAB method, is already given in Paper II. Here we give its functional form applicable to the problems considered in this paper. In Paper II a single preconditioner matrix was sufficient to handle the non-magnetic line transfer problem with PRD. The presence of magnetic field requires the use of domain based PRD matrices, for a better description of the PRD in line scattering. The method requires preconditioner matrices to be defined, that are suitable for each of the frequency domains. We denote the preconditioner matrices by $\hat{\mathcal{M}}^{(i)}$.

$$
\begin{aligned}
& \hat{\mathcal{M}}^{(i)}=\hat{I}-p_{x} \\
& \left.\times \frac{1}{\phi(x)}\left\{\Lambda_{x^{\prime}, \mathrm{II}}^{\star(i)} r_{\mathrm{II}}\left(x, x^{\prime}\right)+\Lambda_{x^{\prime}, \mathrm{III}}^{\star(i)}\right) r_{\mathrm{III}}\left(x, x^{\prime}\right)\right\},
\end{aligned}
$$

where

$$
\Lambda_{x^{\prime}, \mathrm{II}}^{\star(i)}=\oint \frac{d \boldsymbol{\Omega}^{\prime}}{4 \pi} \hat{W} \hat{M}_{\mathrm{II}}^{(i)}(\boldsymbol{B}) \hat{\Psi}\left(\boldsymbol{\Omega}^{\prime}\right) \mathcal{I}\left(\boldsymbol{r}, \boldsymbol{\Omega}^{\prime}, x^{\prime}\right),
$$

and

$\Lambda_{x^{\prime}, \mathrm{III}}^{\star(i)}=\oint \frac{d \boldsymbol{\Omega}^{\prime}}{4 \pi} \hat{W} \hat{M}_{\mathrm{III}}^{(i)}(\boldsymbol{B}) \hat{\Psi}\left(\boldsymbol{\Omega}^{\prime}\right) \mathcal{I}\left(\boldsymbol{r}, \boldsymbol{\Omega}^{\prime}, x^{\prime}\right)$.

Here $\mathcal{I}\left(\boldsymbol{r}, \boldsymbol{\Omega}^{\prime}, x^{\prime}\right)$ is computed using a delta source vector as input. The expressions for the matrices $\hat{M}_{\mathrm{II}}^{(i)}$ and $\hat{M}_{\mathrm{III}}^{(i)}$ in different domains are given in Appendix C. The matrices $\hat{\mathcal{M}}^{(i)}$ are block diagonal. Each block is a full matrix with respect to $x$ and $x^{\prime}$. The matrices $\hat{\mathcal{M}}^{(i)}$ are diagonal with respect to other variables.

\subsection{Computational details}

To calculate the integral in Equation (18) and the formal solution in Equation (22), we need to define quadratures for angles, frequencies and depths.

For all the computations presented in this paper, Carlsson type B angular quadrature with an order $n=8$ is used. All the results are presented in 
this paper for damping parameter $a=10^{-3}$. The number of frequency points required for a given problem depends on the value of $a$ and the optical thickness in the $X, Y$ and $Z$ directions (denoted by $T_{X}, T_{Y}$ and $T_{Z}$ ). A frequency bandwidth satisfying the conditions $\phi\left(x_{\max }\right) T_{X}<<1$, $\phi\left(x_{\max }\right) T_{Y}<<1$ and $\phi\left(x_{\max }\right) T_{Z}<<1$ at the largest frequency point denoted by $x_{\max }$ has been used. We have used a logarithmic frequency grid with a fine spacing in the line core region, and the near wings where the PRD effects are important. We use a logarithmic spacing in the $X, Y$ and $Z$ directions, with a fine griding near the boundaries. We find that with the modern solution methods used in the calculations give sufficiently accurate solutions for 5 spatial points per decade.

Computing time depends on the number of angle, frequency and depth points considered in the calculations and also the machine used for computations. We use the Intel(R) Core(TM) i5 $\mathrm{CPU} 760$ at $2.8 \mathrm{GHz}$ processor running an unparallelized code. For the difficult test case of a semi-infinite 3D atmosphere the computing time is approximately an hour for one iteration. Even for this difficult test case the Pre-BiCG-STAB method needs just 18 iterations to reach a convergence criteria of $10^{-8}$.

\section{RESULTS AND DISCUSSIONS}

In this section we present the results of computations to illustrate broader aspects of the polarized transfer in 1D, 2D and 3D media. We present simple test cases (which can be treated as benchmarks), to show the nature of these solutions. In all the calculations we assume the atmosphere to be isothermal.

We organize our discussions in terms of two effects. One is macroscopic in nature-namely the effect of RT on the Stokes profiles formed in 2D and $3 \mathrm{D}$ media. Another is microscopic in naturenamely the effect of an oriented weak magnetic field on line scattering (Hanle effect). We discuss how these two effects act together on the polarized line formation.

\subsection{The Stokes profiles formed due to res- onance scattering in $2 \mathrm{D}$ and $3 \mathrm{D}$ media}

A discussion on the behavior of Stokes profiles formed in 1D media with PRD scattering can be found in Faurobert (1988) and Nagendra et al. (1999). In Paper II, the nature of profiles in a 2D semi-infinite medium is compared with those formed in 1D semi-infinite medium for CRD and PRD scattering (see Figures 8 and 9 of Paper II). Here we discuss the emergent, spatially averaged $\mathcal{I}$ and $(I, Q / I, U / I)$ in $2 \mathrm{D}$ and $3 \mathrm{D}$ media for PRD scattering.

Figures 5 and 6 show the frequency dependence of the components of emergent, spatially averaged $\mathcal{I}$ in $2 \mathrm{D}$ and $3 \mathrm{D}$ media respectively. The model parameters are, $T_{X}=T_{Y}=T_{Z}=T=2 \times 10^{9}$, $a=10^{-3}, \Gamma_{E} / \Gamma_{R}=10^{-4}, \Gamma_{I} / \Gamma_{R}=10^{-4}$, $\kappa_{c} / \kappa_{l}=10^{-7}$, and $\mu=0.11$. Our choice of collisional parameters represent a situation in which $r_{\text {II }}$ type scattering dominates. Different curves in each panel represent different radiation azimuths $\varphi_{i}(i=1,12)=60,45,30^{\circ}, 300^{\circ}, 315^{\circ}, 330^{\circ}, 120^{\circ}$, $135^{\circ}, 150^{\circ}, 240^{\circ}, 225^{\circ}, 210^{\circ}$.

$I_{0}^{0}$ is the largest of all the components. For the chosen model parameters, all the other non-zero components are of the same order of magnitude. The components $I_{1}^{2, \mathrm{x}}$ and $I_{2}^{2, \mathrm{y}}$ are zero in a $2 \mathrm{D}$ geometry due to symmetry reasons (see Appendix B of Paper II for a proof).

The $\varphi$ dependence of the $\mathcal{I}$ comes from the $\varphi$ dependence of the scattering phase matrix $(\hat{\Psi})$ elements. The spatial distribution of $\mathcal{I}$, on the top surface depends sensitively on the monochromatic optical depths for the ray at these spatial points. This is a transfer effect within the medium, for the chosen ray direction. In the line core frequencies $(x \leq 3)$, the monochromatic optical depths are larger, resulting in a relatively uniform spatial distribution of $\mathcal{I}$ on the top surface. The $\varphi$ dependence appears as either symmetric or antisymmetric with respect to the $X$-axis from which $\varphi$ is measured. Thus the spatial averaging leads to a weak dependence of $\mathcal{I}$ on the azimuth angle $\varphi$. When the averaging is performed over sign changing quantities like the polarization components, it leads to cancellation, resulting in vanishing of these components.

The $\varphi$ dependence of $\mathcal{I}$ in the line wings can be understood by considering the action of the first column elements of the $\hat{\Psi}$ matrix on $I_{0}^{0}$, which is the largest among all the components. The elements of $\hat{\Psi}$ matrix are listed in Appendix D. $I_{0}^{0}$ is independent of $\varphi$ because it is controlled by the element $\Psi_{11}$ which takes a constant value unity. 
Similarly $I_{0}^{2}$ is controlled by $\Psi_{21}$ which is also independent of $\varphi$. However we see a weak $\varphi$ dependence of $I_{0}^{2}$ in the wings, which is due to the coupling of the last 4 components to $I_{0}^{2}$, which are of equal order of magnitude as $I_{0}^{2}$, and are sensitive to the values of $\varphi$. The $\varphi$ dependence of $I_{1}^{2, \mathrm{y}}$ and $I_{2}^{2, \mathrm{x}}$ elements in both $2 \mathrm{D}$ and $3 \mathrm{D}$ geometries is controlled by $\sin \varphi$ and $\cos 2 \varphi$ functions appearing in $\Psi_{41}$ and $\Psi_{51}$ elements respectively. The distribution of angle points $\varphi$ in Carlson B quadrature is such that among the $12 \varphi$ values in the grid, $\sin \varphi$ takes only 6 distinct values, and $\cos 2 \varphi$ takes only 3 distinct values (see Table 1 ). The components $I_{1}^{2, \mathrm{x}}$ and $I_{2}^{2, \mathrm{y}}$ are non-zero in 3D geometry unlike the $2 \mathrm{D}$ case. Their magnitudes are comparable to those of $I_{1}^{2, y}$ and $I_{2}^{2, \mathrm{x}}$. The $\varphi$ dependence of these components are controlled by $\cos \varphi$ and $\sin 2 \varphi$ functions appearing in $\Psi_{31}$ and $\Psi_{61}$ elements. In the far wings, all the components of $\mathcal{I}$ go to their continuum values, as shown in the inset panels of Figures 5 and 6 . In a $1 D$ geometry $I_{0}^{0}$ reaches the value of $B_{\lambda}$ (parameterized as 1 here) in the far wings where the source function is dominated by $B_{\lambda}$. This is because of the fact that the formal solution with $B_{\lambda}$ as source function along a given ray leads to terms of the form $B_{\lambda}\left[1-\exp \left(-\tau_{x, \max }\right)\right]$. In $1 \mathrm{D}$ medium $\tau_{x, \max }=T \kappa_{\text {tot }} / \mu$. This implies that for semiinfinite 1D medium, $\exp \left(-\tau_{x, \max }\right)=0$ so that $I_{0}^{0}=B_{\lambda}$ in the far wings. However in semi-infinite $2 \mathrm{D}$ and $3 \mathrm{D}$ media the distances traveled by the rays in a given direction at different spatial points on the top surface are not always the same and therefore $\exp \left(-\tau_{x, \max }\right)$ is not always zero unlike the $1 \mathrm{D}$ case. Further the radiation drops sharply near the edges due to finiteness of the boundaries. Therefore when we perform spatial averaging of emergent $I_{0}^{0}$ over such different spatial points on the top surface of a $2 \mathrm{D}$ medium (which is actually a line), $I_{0}^{0}$ will take a value smaller than $B_{\lambda}$. For a similar reason (averaging over a plane) the value of $I_{0}^{0}$ in the far wings in a 3D medium becomes even smaller than the value in a $2 \mathrm{D}$ medium. All other components reach zero in the far wings because the radiation is unpolarized in the far wings (because of an unpolarized continuum).

The way in which the components of $\mathcal{I}$ depend on $\varphi$ is different in 2D and 3D geometries (compare Figures 5 and 6 ). This is a direct effect of spatial averaging. In a $2 \mathrm{D}$ medium, spatial averaging of the profiles is performed over the line $\left(Y, Z_{\max }\right)$ marked in Figure 1, whereas in a 3D medium the averaging is performed over the plane $\left(X, Y, Z_{\max }\right)$ marked in Figure 1. The 2D spatial averaging actually samples only a part of the plane considered for averaging in a 3D medium. Also, 2D geometry has an implicit assumption of front-back symmetry of the polarized radiation field with respect to the infinite $X$ axis in the non-magnetic case, namely

$$
\begin{aligned}
& I(\boldsymbol{r}, \theta, \varphi, x)=I(\boldsymbol{r}, \theta, \pi-\varphi, x), \\
& I(\boldsymbol{r}, \theta, \pi+\varphi, x)=I(\boldsymbol{r}, \theta, 2 \pi-\varphi, x), \\
& Q(\boldsymbol{r}, \theta, \varphi, x)=Q(\boldsymbol{r}, \theta, \pi-\varphi, x), \\
& Q(\boldsymbol{r}, \theta, \pi+\varphi, x)=Q(\boldsymbol{r}, \theta, 2 \pi-\varphi, x), \\
& U(\boldsymbol{r}, \theta, \varphi, x)=-U(\boldsymbol{r}, \theta, \pi-\varphi, x), \\
& U(\boldsymbol{r}, \theta, \pi+\varphi, x)=-U(\boldsymbol{r}, \theta, 2 \pi-\varphi, x), \\
& \theta \in[0, \pi], \varphi \in[0, \pi / 2] .
\end{aligned}
$$

See Appendix B of Paper II for a proof of Equation (26). However no such assumptions are involved in $3 \mathrm{D}$ geometry.

Figures 7(a), (b) and (c) show $I, Q / I, U / I$ profiles in non-magnetic $1 \mathrm{D}, 2 \mathrm{D}$ and $3 \mathrm{D}$ media. Intensity $I$ decreases monotonically from $1 \mathrm{D}$ to the $3 \mathrm{D}$ case, because of the leaking of radiation through the finite boundaries in the lateral directions which is specific to $\mathrm{RT}$ in $2 \mathrm{D}$ and $3 \mathrm{D}$ geometries. In panels (b) and (c), different curves represent different $\varphi$ values. Only one curve is shown in panel (a), because of the axi-symmetry of the radiation field in the $1 \mathrm{D}$ medium. For the same reason, $|U / I|_{1 \mathrm{D}}=0$. The $\varphi$ dependence of $|Q / I|_{2 \mathrm{D}, 3 \mathrm{D}}$ and $|U / I|_{2 \mathrm{D} .3 \mathrm{D}}$ directly follow from those of the components of $\mathcal{I}$ shown in Figures 5 and 6, and their combinations (see Appendix A in this paper where we list the formulae used to construct the Stokes vector $(I, Q, U)^{T}$ from the irreducible components of $\mathcal{I})$. At the line center, $[U / I]_{2 \mathrm{D}, 3 \mathrm{D}} \sim 0$. This is because $U / I$ is zero in large parts of the top surface and the positive and negative values of $U / I$ at $x=0$ are nearly equally distributed in a narrow region near the edges. A spatial averaging of such a distribution leads to cancellation giving a net value of $U / I$ approaching zero. This is not the case in wing frequencies of the $U / I$ profile (see discussions in Section 6.3 for spatial distribution of $Q / I$ and $U / I)$. 


\subsection{The Stokes profiles in $2 \mathrm{D}$ and $3 \mathrm{D}$ me- dia in the presence of a magnetic field}

Figures 8, 9 and 10 show all the 6 components of $\mathcal{I}$ in magnetized 1D, 2D and 3D media respectively. The vector magnetic field $\boldsymbol{B}$ is represented by $\left(\Gamma, \theta_{B}, \chi_{B}\right)=\left(1,90^{\circ}, 68^{\circ}\right)$. The corresponding non-magnetic components are shown as thin solid lines. Different line types in Figures 9 and 10 correspond to different $\varphi$. The irreducible components in 1D geometry are cylindrically symmetrical, even when there is an oriented magnetic field. Therefore there is only one curve in each panel in Figure 8. When $\boldsymbol{B}=0$ the 4 components $I_{1,2}^{2 \mathrm{x}, \mathrm{y}}$ become zero due to axi-symmetry in $1 \mathrm{D}$ geometry (Figure 8). These components take non-zero values in the line core when $\boldsymbol{B} \neq 0$. The magnitudes of $I_{0}^{0}$ and $I_{0}^{2}$ monotonically decrease from $1 \mathrm{D}$ to $3 \mathrm{D}$. In the $2 \mathrm{D}$ case, the 2 components which were zero when $\boldsymbol{B}=0$, take non-zero values in the line core, when $\boldsymbol{B} \neq 0$. Unlike $1 \mathrm{D}$ geometry in $2 \mathrm{D}$ and 3D geometries, a non-zero $\boldsymbol{B}$ causes the last 4 components to become sensitive to $\varphi$. The components $I_{1}^{2 \mathrm{y}}$ in $2 \mathrm{D}$ and $I_{1}^{2 \mathrm{x}}$ and $I_{1}^{2 \mathrm{y}}$ in $3 \mathrm{D}$ remain almost unaffected by $\boldsymbol{B}$. This behavior is particular to the present choice of $\boldsymbol{B}$. For a different choice of $\boldsymbol{B}$, the behavior of the 6 components may differ from what is shown in these figures. In all the geometries, the components go to their non-magnetic (Rayleigh scattering) values in the wings, because the Hanle effect operates only in the line core region.

Figures 11(a), (b) and (c) show spatially averaged $I, Q / I, U / I$ in $1 \mathrm{D}, 2 \mathrm{D}$ and $3 \mathrm{D}$ geometries respectively. Due to the finiteness of the boundaries in $2 \mathrm{D}$ and $3 \mathrm{D}$ media the value of spatially averaged $I$ decreases monotonically from $1 \mathrm{D}$ to $3 \mathrm{D}$. The dependence of $Q / I$ and $U / I$ on $\varphi$ in $1 \mathrm{D}$ medium is purely due to the $\varphi$ dependence coming from the formulae used to convert $\mathcal{I}$ to $I, Q$ and $U$ (see Appendix A). In 2D and 3D media, the $\varphi$ dependence comes from both, the $\varphi$ dependence of the respective components of $\mathcal{I}$, and also the above mentioned conversion formulae. The magnitudes of $Q / I$ and $U / I$ decrease in 2D and 3D geometries due to the spatial averaging process. The wings of $Q / I$ and $U / I$ in $1 \mathrm{D}$ are insensitive to $\varphi$ due to the inherent axi-symmetry. In $2 \mathrm{D}$ they become more sensitive to $\varphi$ values. Again they become weakly sensitive to $\varphi$ in 3D geometry. These differences in sensitivities of $Q / I, U / I$ to the azimuth angle $\varphi$ in $2 \mathrm{D}$ and $3 \mathrm{D}$ geometries is due to the way in which the spatial averaging is performed in these geometries (see discussions above Equation (26)).

\subsubsection{Polarization diagrams in $1 D$ and $2 D$ me- dia}

In Figure 12 we show polarization diagrams (see e.g., Stenflo 1994), which are plots of $Q / I$ versus $U / I$ for a given value of frequency $x$, ray direction $(\mu, \varphi)$, and varying the field parameters two out of three at a time. We take $\Gamma=1$, and vary $\theta_{B}$ and $\chi_{B}$ values. For the $2 \mathrm{D}$ case we show spatially averaged quantities.

For $x=0$, the shapes of closed curves (loops) in the polarization diagrams are the same in both $1 \mathrm{D}$ and $2 \mathrm{D}$ cases. When compared to the loops in 1D, the sizes of the loops in $2 \mathrm{D}$ are smaller by about $1 \%$ in the magnitudes of $Q / I$ and $U / I$, which is due to spatial averaging.

For $x=2.5$, the shapes of the the loops in 2D are quite different from those for 1D. For e.g., the solid curve in panel $(d)$ is narrower than the one in panel (b) which correspond to $\theta_{B}=30^{\circ}$. On the other hand, the dash-triple-dotted curve in panel (d) is broader than the one in panel (b), which correspond to $\theta_{B}=120^{\circ}$. The orientation of a given loop with respect to the vertical line $(Q / I=$ $0)$ is a measure of the sensitivity of $(Q / I, U / I)$ to the field orientation $\theta_{B}$. The size of a loop is a measure of the sensitivity of $(Q / I, U / I)$ to the field azimuth $\chi_{B}$. The values of $|Q / I|_{2 D}$ and $|U / I|_{2 D}$ can be larger or smaller than $|Q / I|_{1 D}$ and $|U / I|_{1 D}$ for $x=2.5$. The sensitivity of the line wing $(x=$ $2.5)$ polarization to $\left(\theta_{B}, \chi_{B}\right)$ is different in $1 \mathrm{D}$ and $2 \mathrm{D}$ geometries, when compared to the sensitivity of line center $(x=0)$ polarization. This is because at $x=0$ we sample mainly the outermost layers of the semi-infinite media. At $x=2.5$ we actually sample internal inhomogeneities of the radiation field in $(Y, Z)$ directions in the $2 \mathrm{D}$ case, and only those in the $Z$ direction, in the $1 \mathrm{D}$ case. We have noticed that the spatial distribution of $Q / I, U / I$ at $x=0$ is relatively more homogeneous, than at $x=2.5$ (see figures and discussions in Section 6.3 for spatial distribution of $Q / I$ and $U / I)$. 


\subsection{The spatial variation of emergent $(Q / I, U / I)$ in a $3 \mathbf{D}$ medium}

In Figure 13 we show surface plots of $Q / I$ and $U / I$ formed in a $3 \mathrm{D}$ media. The region chosen for showing the spatial distribution is the top surface plane $\left(X, Y, Z_{\max }\right)$.

Figures 13(a), (b) demonstrate purely the effects of multi-D geometry on the $(Q / I, U / I)$ profiles. In Figure 13(a) $Q / I$ shows a homogeneous distribution at the interiors of the top surface (away from the boundaries) approaching a constant value $(\sim-3.6 \%)$. Large parts of the top surface contribute to the negative values of $Q / I$ and only a narrow region near the edges contribute to positive values. The magnitudes of $Q / I$ sharply raise near the edges. This is due to the finite boundaries of the 3D medium. Maximum value of $|Q / I|$ in these figures is $\sim 6 \%$. In Figure 13(b) $U / I$ is nearly zero at the interiors of the top surface. Near the edges, the values of $U / I$ sharply raise and $|U / I|$ takes a maximum value of $\sim 20 \%$.

Figures 13(c), (d) demonstrate the effects of magnetic field on the $(Q / I, U / I)$ profiles. The magnetic field vector is represented by $\boldsymbol{B}=\left(\Gamma, \theta_{B}, \chi_{B}\right)=\left(1,30^{\circ}, 68^{\circ}\right)$. The nature of homogeneity at the interior and sharp raise near the edges of the 3D surface, in the values of $Q / I$ and $U / I$ remain similar in both the magnetic and nonmagnetic cases. An important effect of $\boldsymbol{B}$ is to significantly change the values of $Q / I$ and $U / I$ with respect to their non-magnetic values. $|Q / I|$ values are slightly reduced at the interior and $Q / I$ now becomes $-2.3 \%$. Near the edges $|Q / I|$ is significantly enhanced and takes a maximum value of $15 \%$. The interior values of $|U / I|$ continue to be nearly zero. The $|U / I|$ is reduced at different rates near different edges. Now the maximum value of $|U / I|$ is $17 \%$. We note that in $1 \mathrm{D}$ geometry, for $\mu=0.11$, any magnetic field configuration always causes a decrease in $|Q / I|$ and a fresh generation of $|U / I|$ with respect to the non-magnetic values.

Figures 13(e), (f) demonstrate the effects of PRD on the $(Q / I, U / I)$ profiles. For this purpose we have chosen a wing frequency $x=5$. The spatial distribution of $Q / I$ and $U / I$ is highly inhomogeneous at the wing frequencies. This effect can be easily seen by comparing Figure 13(a) which exhibits large spatial homogeneity for $x=0$, with Figure 13(e) which exhibits large spatial inhomo- geneity for $x=5$. For $x=0$, the optical depth of the medium is large and therefore the radiation field in the line core becomes homogeneous over large volumes of the cube. The spatial inhomogeneity of the $Q / I$ at $x=5$ is actually caused by the the nature of PRD function used in our computations (which is dominated by the $r_{\mathrm{II}}$ function). Due to the frequency coherent nature of $r_{\mathrm{II}}$, the photons scattered in the wings get decoupled from the line core radiation field. As the optical depth of the medium in the line wings is smaller than in the line core, the wing radiation field becomes more inhomogeneous and more polarized. Same arguments are valid for the inhomogeneous distribution of $U / I$ on the top surface of the $3 \mathrm{D}$ cube. This can be seen by comparing Figure 13(b) with Figure 13(f). We recall that under the assumption of CRD, the values of $Q / I$ and $U / I$ are zero in the line wings (see Figure 9 of Paper II for a comparison of emergent, spatially averaged $Q / I, U / I$ profiles for CRD and PRD in a multi-D medium). The sharp increase in magnitudes of $Q / I$ and $U / I$ near the edges is larger for $x=5$ when compared to those for $x=0$. Maximum value of $|Q / I|$ is now $10 \%$ and that of $|U / I|$ is $40 \%$.

In Figure 14 we show spatial distribution of $I$, $Q / I$ and $U / I$ on the top surface of two different kinds of $3 \mathrm{D}$ media. Here we have chosen $\boldsymbol{B}=0$ which is equivalent to the choice of a vertical magnetic field parallel to the $Z$ axis (because, for this field geometry the Hanle effect goes to its nonmagnetic Rayleigh scattering limit). In view of the possible applications, we consider a cuboid with $T_{X}=T_{Y}=2 \times 10^{6}, T_{Z}=20$ in the left panels $(\mathrm{a}, \mathrm{b}, \mathrm{c})$ and a cuboid with $T_{X}=T_{Y}=20$, $T_{Z}=2 \times 10^{6}$ in the right panels (d, e, f). They represent respectively a sheet and a rod like structure. For the chosen optical thickness configurations, the radiative transfer effects are mainly restricted to the line core $(x \leq 3)$ for the ray emerging from the top surface. We show the results for $x=3$ (in the left panels) and $x=1$ (in the right panels), the frequencies for which the magnitudes of $Q / I$ and $U / I$ reach their maximum values.

In Figures 14(a) and (d) the intensities reach saturation values in the interiors of the top surface and drop to zero at two of the visible boundaries (where a boundary condition of zero intensity is imposed for our chosen ray emerging at the top surface). 
In Figures 14(b) and (c) we see that $Q / I$ and $U / I$ take values $\leq 1 \%$ everywhere on the top surface. The magnitude of $Q / I$ and $U / I$ for this case are relatively less than those for the semiinfinite 3D atmospheres (compare with Figure 13). This can be understood using the following arguments. We are showing the results for a ray with $(\mu, \varphi)=\left(0.11,60^{\circ}\right)$ emerging from the top surface. The top surface for this figure refers to $\tau_{Z}=0$ where $\tau_{Z}$ is the optical depth measured inwards in the $Z$ direction. Using equations given in Appendix A we can write approximate expressions for $Q$ and $U$ at the top surface as

$$
\begin{aligned}
& Q\left(\mu=0.11, \varphi=60^{\circ}, x\right) \approx \\
& \frac{-3}{2 \sqrt{2}} I_{0}^{2}\left(\mu=0.11, \varphi=60^{\circ}, x\right), \\
& U\left(\mu=0.11, \varphi=60^{\circ}, x\right) \approx \\
& \frac{3}{2} I_{1}^{2, \mathrm{x}}\left(\mu=0.11, \varphi=60^{\circ}, x\right) \\
& +\frac{\sqrt{3}}{2} I_{1}^{2, \mathrm{y}}\left(\mu=0.11, \varphi=60^{\circ}, x\right) .
\end{aligned}
$$

$I_{0}^{2}$ is controlled by the element $\Psi_{21}=3 \cos ^{2} \theta-1$ (see Appendix D) which appears in the scattering integral for $S_{0}^{2}$. The factor $\Psi_{21}=3 \cos ^{2} \theta-1$ represents the probability of scattering of photons incident from the direction $\theta$. For $\theta=0^{\circ}$ or $\theta=180^{\circ}$ (vertical incidence) $\Psi_{21}$ is larger in magnitude compared to the cases $\theta=90^{\circ}$ or $\theta=270^{\circ}$ (lateral incidence). For $T_{Z}=20$ the medium is effectively optically thin (because $\epsilon T_{Z}<<1$ ) in the $Z$ direction, and therefore photons easily escape in this direction. Thus there are smaller number of photons for incidence along the vertical direction when compared to the effectively thick case. For $T_{Z}=2 \times 10^{6}$ or $T_{Z}=2 \times 10^{9}$ the medium is effectively optically thick (because $\epsilon T_{Z}>>1$ ) in the $Z$ direction and therefore leaking of photons in this direction is reduced when compared to the case of $T_{Z}=20$. In this way, for large values of $T_{Z}$ the probability of photons to be incident in the vertical direction is large. Therefore, as $T_{Z}$ increases the values of $I_{0}^{2}$ and hence $Q / I$ increase.

For the chosen line of sight, Stokes $U$ is generated mainly by $I_{1}^{2, \mathrm{x}}$ and $I_{1}^{2, \mathrm{y}}$. They are controlled by $\Psi_{31}$ and $\Psi_{41}$ elements (see Appendix D) both of which depend on the factor $\sin 2 \theta$. This implies that $\Psi_{31}$ and $\Psi_{41}$ are zero for both vertical and lateral incidence of photons. These elements become larger when the incidence is predominant in the direction of $\theta=45^{\circ}$ or $\theta=135^{\circ}$. Using similar arguments as above we can understand the increase in the values of $U / I$ with increasing values of $T_{Z}$.

The spatial distribution of $Q / I$ and $U / I$ is inhomogeneous in both left and right panels for the chosen core frequencies, in contrast to the homogeneous distribution observed for semi-infinite 3D atmospheres. The extent of inhomogenity is larger for the left panels which correspond to smaller $T_{Z}$ value than for the right panels. The spatial inhomogenity could also occur due to different optical thicknesses along the 3 spatial directions leading to different number of scatterings in the 3 directions (unlike the case of Figure 13 where $\left.T_{X}=T_{Y}=T_{Z}\right)$. In other words, the inhomogeneities in $Q / I$ and $U / I$ can also be caused by a differential leaking of radiation in the $X, Y$ and $Z$ directions.

\section{CONCLUSIONS}

This paper is dedicated to certain extensions of our previous works (Paper I and Paper II) on polarized RT in multi-D media with PRD.

First, we present a generalization of the Stokes vector decomposition technique developed in $\mathrm{Pa}$ per I, to include the magnetic fields (Hanle effect).

Secondly, we generalize to the magnetic 3D RT, the efficient iterative method called the Pre-BiCGSTAB developed in Paper II for the non-magnetic 2D RT.

Thirdly, we use the more efficient $2 \mathrm{D}$ and $3 \mathrm{D}$ short characteristics formal solutions, with appropriate generalizations to the present context. With the linear formal solver used in Paper I, practically it is difficult to compute the solutions in semi-infinite media. It is not the case with the short characteristics former solution method. Indeed, the solutions presented in this paper for the difficult case of semi-infinite media, prove this fact.

We present several benchmark solutions computed using the code, with all the above mentioned generalizations. The main results of these solutions are the following.

The emergent $(Q / I, U / I)$ profiles in $1 \mathrm{D}$ media and the emergent, spatially averaged $(Q / I, U / I)$ profiles in 2D and 3D media differ significantly, 
both in non-magnetic and magnetic cases. The differences are more pronounced in the wings of the $(Q / I, U / I)$ profiles. The differences between the emergent, spatially averaged $(Q / I, U / I)$ profiles in $2 \mathrm{D}$ and $3 \mathrm{D}$ media are negligible in $Q / I$, but noticeable in $U / I$.

In the non-magnetic case, at line center, the spatial distribution of $Q / I$ and $U / I$ is homogeneous in the interior of the top surface, but sharply raise near the edges. This is purely a multi-D geometric effect. The presence of a magnetic field modifies this distribution by causing a depolarization (decrease in the magnitude) or re-polarization (increase in the magnitude) of $Q / I$ and $U / I$. This is a natural consequence of the Hanle effect. In the line wing frequencies, magnetic and non-magnetic spatial distributions look the same, as Hanle effect is confined to the line core. However, the spatial distribution in the line wing frequency is more inhomogeneous, and the sharp raise of $Q / I$ and $U / I$ near the edges is more enhanced, as compared to those at the line center. This behavior at line wings is mainly due to the PRD effects. These characteristics are not noticeable if the CRD assumption is used in line formation studies.

We have developed efficient techniques to solve polarized RT in multi-D media with PRD as the scattering mechanism. In future, we try to apply these methods to understand the linear polarization observed in the spatially resolved structures on the Sun.

We would like to thank Prof. H. Frisch for useful suggestions which helped to improve the manuscript. We thank Dr. Sampoorna for useful discussions. 


\section{A. EXPANSION OF STOKES PARAMETERS INTO THE IRREDUCIBLE COMPO- NENTS}

The Stokes parameters and the irreducible Stokes vector are related through the following expressions. They are already given in Frisch (2007). However we present these expressions here for an easy reference.

$$
\begin{aligned}
& I(\boldsymbol{r}, \boldsymbol{\Omega}, x)=I_{0}^{0}+\frac{1}{2 \sqrt{2}}\left(3 \cos ^{2} \theta-1\right) I_{0}^{2} \\
& -\sqrt{3} \cos \theta \sin \theta\left(I_{1}^{2, \mathrm{x}} \cos \varphi-I_{1}^{2, \mathrm{y}} \sin \varphi\right) \\
& +\frac{\sqrt{3}}{2}\left(1-\cos ^{2} \theta\right)\left(I_{2}^{2, \mathrm{x}} \cos 2 \varphi-I_{2}^{2, \mathrm{y}} \sin 2 \varphi\right) \\
& Q(\boldsymbol{r}, \boldsymbol{\Omega}, x)=-\frac{3}{2 \sqrt{2}}\left(1-\cos ^{2} \theta\right) I_{0}^{2} \\
& -\sqrt{3} \cos \theta \sin \theta\left(I_{1}^{2, \mathrm{x}} \cos \varphi-I_{1}^{2, \mathrm{y}} \sin \varphi\right) \\
& -\frac{\sqrt{3}}{2}\left(1+\cos ^{2} \theta\right)\left(I_{2}^{2, \mathrm{x}} \cos 2 \varphi-I_{2}^{2, \mathrm{y}} \sin 2 \varphi\right) \\
& U(\boldsymbol{r}, \boldsymbol{\Omega}, x)=\sqrt{3} \sin \theta\left(I_{1}^{2, \mathrm{x}} \sin \varphi+I_{1}^{2, \mathrm{y}} \cos \varphi\right) \\
& +\sqrt{3} \cos \theta\left(I_{2}^{2, \mathrm{x}} \sin 2 \varphi+I_{2}^{2, \mathrm{y}} \cos 2 \varphi\right) .
\end{aligned}
$$

The irreducible components in the above equations also depend on $\boldsymbol{r}, \boldsymbol{\Omega}, x$ and $\boldsymbol{B}$.

\section{B. THE REDISTRIBUTION MATRICES IN THE IRREDUCIBLE TENSORIAL FORM}

In this paper we use the redistribution matrices defined under the approximation level III of Bommier (1997b). The expressions listed below are already given in Bommier (1997b). We give them here for the sake of completeness. The branching ratios (see Bommier 1997b) are given by

$$
\begin{gathered}
\alpha=\frac{\Gamma_{R}}{\Gamma_{R}+\Gamma_{E}+\Gamma_{I}}, \\
\beta^{(K)}=\frac{\Gamma_{R}}{\Gamma_{R}+D^{(K)}+\Gamma_{I}},
\end{gathered}
$$

with $D^{(0)}=0$ and $D^{(2)}=c \Gamma_{E}$, where $c$ is a constant, taken to be 0.379 (see Faurobert-Scholl 1992).

The Hanle $\Gamma_{B}$ coefficient (see Bommier 1997b) takes two different forms, namely

$$
\Gamma_{B}=\Gamma_{K}^{\prime}=\beta^{(K)} \Gamma, \quad \Gamma_{B}=\Gamma^{\prime \prime}=\alpha \Gamma,
$$

with

$$
\Gamma=g_{J} \frac{2 \pi e B}{2 m_{e} \Gamma_{R}}
$$

where $e B / 2 m_{e}$ is the Larmor frequency of the electron in the magnetic field (with $e$ and $m_{e}$ being the charge and mass of the electron). Here $B$ is the magnetic field strength. The expressions for the redistribution 
matrices given in Bommier (1997b) involve a cut-off frequency $v_{c}(a)$, which is given by the solution of the equation

$$
\frac{1}{\sqrt{\pi}} e^{-v^{2}}=\frac{a}{\pi} \frac{1}{v^{2}+a^{2}}
$$

and a constant $z=2 \sqrt{2}+2$ coming from the angle-averaging process.

If

$z v_{c}(a)\left|x^{\prime}\right|-\left(x^{2}+x^{\prime 2}\right)<(z-1) v_{c}^{2}(a)$ and

$z v_{c}(a)|x|-\left(x^{2}+x^{2}\right)<(z-1) v_{c}^{2}(a)$ and

$\left|x^{\prime}\right|<\sqrt{2} v_{c}(a)$ and $|x|<\sqrt{2} v_{c}(a)$,

then domain 1 :

$$
\begin{aligned}
& P_{Q, \mathrm{III}}^{K}\left(j, \boldsymbol{\Omega}^{\prime}, \boldsymbol{B}\right)=\sum_{Q^{\prime}}\left\{\beta^{(K)} \mathcal{M}_{Q Q^{\prime}}^{K}\left(\boldsymbol{B} ; \Gamma_{K}^{\prime}\right)\right. \\
& \left.-\alpha \mathcal{M}_{Q Q^{\prime}}^{K}\left(\boldsymbol{B} ; \Gamma^{\prime \prime}\right)\right\}(-1)^{Q^{\prime}} \mathcal{T}_{-Q^{\prime}}^{K}\left(j, \boldsymbol{\Omega}^{\prime}\right) \\
& =\sum_{Q^{\prime}} \overline{\mathcal{M}}_{Q Q^{\prime}, \mathrm{III}}^{K(1)}(\boldsymbol{B})\left(\mathcal{T}_{Q^{\prime}}^{K}\right)^{*}\left(j, \boldsymbol{\Omega}^{\prime}\right) .
\end{aligned}
$$

elseif

$\left|x^{\prime}\right|<v_{c}(a)$ or $|x|<v_{c}(a)$,

then domain 2 :

$$
\begin{aligned}
& P_{Q, \mathrm{III}}^{K}\left(j, \boldsymbol{\Omega}^{\prime}, \boldsymbol{B}\right)=\left[\beta^{(K)}-\alpha\right] \\
& \times \sum_{Q^{\prime}} \mathcal{M}_{Q Q^{\prime}}^{K}\left(\boldsymbol{B} ; \Gamma_{K}^{\prime}\right)(-1)^{Q^{\prime}} \mathcal{T}_{-Q^{\prime}}^{K}\left(j, \boldsymbol{\Omega}^{\prime}\right), \\
& =\sum_{Q^{\prime}} \overline{\mathcal{M}}_{Q Q^{\prime}, \mathrm{III}}^{K(2)}(\boldsymbol{B})\left(\mathcal{T}_{Q^{\prime}}^{K}\right)^{*}\left(j, \boldsymbol{\Omega}^{\prime}\right) .
\end{aligned}
$$

else domain 3 :

$$
\begin{aligned}
& P_{Q, \mathrm{III}}^{K}\left(j, \boldsymbol{\Omega}^{\prime}, \boldsymbol{B}\right)=\left[1-\alpha / \beta^{(K)}\right]\left\{\left[\beta^{(K)}-\alpha\right]\right. \\
& \times \sum_{Q^{\prime}} \mathcal{M}_{Q Q^{\prime}}^{K}\left(\boldsymbol{B} ; \Gamma_{K}^{\prime}\right)(-1)^{Q^{\prime}} \mathcal{T}_{-Q^{\prime}}^{K}\left(j, \boldsymbol{\Omega}^{\prime}\right) \\
& \left.+\alpha \sum_{Q^{\prime}}(-1)^{Q^{\prime}} \mathcal{T}_{-Q^{\prime}}^{K}\left(j, \boldsymbol{\Omega}^{\prime}\right)\right\} \\
& =\sum_{Q^{\prime}} \overline{\mathcal{M}}_{Q Q^{\prime}, \mathrm{III}}^{K(B)}(\boldsymbol{B})\left(\mathcal{T}_{Q^{\prime}}^{K}\right)^{*}\left(j, \boldsymbol{\Omega}^{\prime}\right) .
\end{aligned}
$$

endif. If

$x\left(x+x^{\prime}\right)<2 v_{c}^{2}(a) \quad$ and $\quad x^{\prime}\left(x+x^{\prime}\right)<2 v_{c}^{2}(a)$, 
then domain 4 :

$$
\begin{aligned}
& P_{Q, \mathrm{II}}^{K}\left(j, \boldsymbol{\Omega}^{\prime}, \boldsymbol{B}\right) \\
& =\alpha \sum_{Q^{\prime}} \mathcal{M}_{Q Q^{\prime}}^{K}\left(\boldsymbol{B} ; \Gamma^{\prime \prime}\right)(-1)^{Q^{\prime}} \mathcal{T}_{-Q^{\prime}}^{K}\left(j, \boldsymbol{\Omega}^{\prime}\right) \\
& =\sum_{Q^{\prime}} \overline{\mathcal{M}}_{Q Q^{\prime}, \mathrm{II}}^{K(4)}(\boldsymbol{B})\left(\mathcal{T}_{Q^{\prime}}^{K}\right)^{*}\left(j, \boldsymbol{\Omega}^{\prime}\right)
\end{aligned}
$$

else domain 5 :

$$
\begin{aligned}
& P_{Q, \mathrm{II}}^{K}\left(j, \boldsymbol{\Omega}^{\prime}, \boldsymbol{B}\right)=\alpha \sum_{Q^{\prime}}(-1)^{Q^{\prime}} \mathcal{T}_{-Q^{\prime}}^{K}\left(j, \boldsymbol{\Omega}^{\prime}\right), \\
& =\sum_{Q^{\prime}} \overline{\mathcal{M}}_{Q Q^{\prime}, \mathrm{II}}^{K(\boldsymbol{B})}(\boldsymbol{B})\left(\mathcal{T}_{Q^{\prime}}^{K}\right)^{*}\left(j, \boldsymbol{\Omega}^{\prime}\right) .
\end{aligned}
$$

endif.

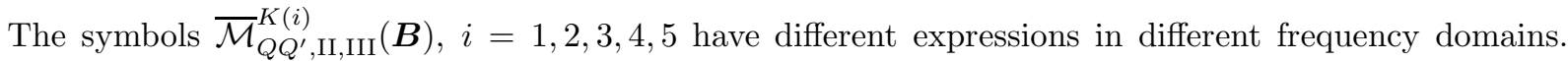
They implicitly contain the respective branching ratios and the Hanle $\Gamma$ parameter depending upon the domain.

\section{THE REDISTRIBUTION MATRICES IN THE MATRIX FORM}

We introduce the diagonal matrices

$$
\hat{\alpha}=\alpha \hat{E}
$$

with $\hat{E}$ the identity matrix,

$$
\begin{aligned}
& \hat{\beta}=\operatorname{diag}\left\{\beta^{(0)}, \beta^{(2)}, \beta^{(2)}, \beta^{(2)}, \beta^{(2)}, \beta^{(2)}\right\}, \\
& \hat{\mathcal{F}}=\operatorname{diag}\left\{1-\frac{\alpha}{\beta^{(0)}}, 1-\frac{\alpha}{\beta^{(2)}}, 1-\frac{\alpha}{\beta^{(2)}},\right. \\
& \left.1-\frac{\alpha}{\beta^{(2)}}, 1-\frac{\alpha}{\beta^{(2)}}, 1-\frac{\alpha}{\beta^{(2)}}\right\} .
\end{aligned}
$$

The real matrices $\hat{M}_{\mathrm{II}}^{(i)}(\boldsymbol{B})$ and $\hat{M}_{\mathrm{III}}^{(i)}(\boldsymbol{B})$ have following expressions in different domains.

In domain 1:

$$
\hat{M}_{\mathrm{III}}^{(1)}(\boldsymbol{B})=\left\{\hat{\beta} \hat{M}\left(\boldsymbol{B}, \Gamma_{2}^{\prime}\right)-\hat{\alpha} \hat{M}\left(\boldsymbol{B}, \Gamma^{\prime \prime}\right)\right\} .
$$

In domain 2:

$$
\hat{M}_{\mathrm{III}}^{(2)}(\boldsymbol{B})=\left\{[\hat{\beta}-\hat{\alpha}] \hat{M}\left(\boldsymbol{B}, \Gamma_{K}^{\prime}\right)\right\} .
$$


In domain 3:

$$
\hat{M}_{\mathrm{III}}^{(3)}(\boldsymbol{B})=\hat{\mathcal{F}}\left\{[\hat{\beta}-\hat{\alpha}] \hat{M}\left(\boldsymbol{B}, \Gamma_{2}^{\prime}\right)+\hat{\alpha}\right\} .
$$

In domain 4:

$$
\hat{M}_{\mathrm{II}}^{(4)}(\boldsymbol{B})=\hat{\alpha} \hat{M}\left(\boldsymbol{B}, \Gamma^{\prime \prime}\right) .
$$

In domain 5:

$$
\hat{M}_{\mathrm{II}}^{(5)}(\boldsymbol{B})=\hat{\alpha} .
$$

\section{The scattering phase matrix in real form in the reduced basis}

The elements of the matrix $\hat{\Psi}$ are already given in Appendix A of Paper I. However we have found that there were some typographical errors there. We give here the elements again, correcting those typographical errors.

$$
\hat{\Psi}^{r}=\left(\begin{array}{cccccc}
\Psi_{11} & \Psi_{12} & \Psi_{13} & \Psi_{14} & \Psi_{15} & \Psi_{16} \\
\Psi_{12} & \Psi_{22} & \Psi_{23} & \Psi_{24} & \Psi_{25} & \Psi_{26} \\
\frac{1}{2} \Psi_{13} & \frac{1}{2} \Psi_{23} & \Psi_{33} & \Psi_{34} & \Psi_{35} & \Psi_{36} \\
\frac{1}{2} \Psi_{14} & \frac{1}{2} \Psi_{24} & \Psi_{34} & \Psi_{44} & \Psi_{45} & \Psi_{46} \\
\frac{1}{2} \Psi_{15} & \frac{1}{2} \Psi_{25} & \Psi_{35} & \Psi_{45} & \Psi_{55} & \Psi_{56} \\
\frac{1}{2} \Psi_{16} & \frac{1}{2} \Psi_{26} & \Psi_{36} & \Psi_{46} & \Psi_{56} & \Psi_{66}
\end{array}\right),
$$


where the distinct matrix elements are:

$$
\begin{aligned}
& \Psi_{11}=1 ; \quad \Psi_{12}=\frac{1}{2 \sqrt{2}}\left(3 \cos ^{2} \theta-1\right) ; \\
& \Psi_{13}=-\frac{\sqrt{3}}{2} \sin 2 \theta \cos \varphi ; \quad \Psi_{14}=\frac{\sqrt{3}}{2} \sin 2 \theta \sin \varphi ; \quad \Psi_{15}=\frac{\sqrt{3}}{2} \sin ^{2} \theta \cos 2 \varphi ; \\
& \Psi_{16}=-\frac{\sqrt{3}}{2} \sin ^{2} \theta \sin 2 \varphi ; \quad \Psi_{22}=\frac{1}{4}\left(9 \cos ^{4} \theta-12 \cos ^{2} \theta+5\right) ; \\
& \Psi_{23}=\frac{\sqrt{3}}{4 \sqrt{2}} \sin 2 \theta(1-3 \cos 2 \theta) \cos \varphi ; \quad \Psi_{24}=-\frac{\sqrt{3}}{4 \sqrt{2}} \sin 2 \theta(1-3 \cos 2 \theta) \sin \varphi ; \\
& \Psi_{25}=\frac{\sqrt{3}}{2 \sqrt{2}} \sin ^{2} \theta\left(1+3 \cos ^{2} \theta\right) \cos 2 \varphi ; \quad \Psi_{26}=-\frac{\sqrt{3}}{2 \sqrt{2}} \sin ^{2} \theta\left(1+3 \cos ^{2} \theta\right) \sin 2 \varphi ; \\
& \Psi_{33}=\frac{3}{4} \sin ^{2} \theta\left[\left(1+2 \cos ^{2} \theta\right)-\left(1-2 \cos ^{2} \theta\right) \cos 2 \varphi\right] \\
& \Psi_{34}=\frac{3}{4} \sin ^{2} \theta\left(1-2 \cos ^{2} \theta\right) \sin 2 \varphi ; \quad \Psi_{35}=\frac{3}{16} \sin 2 \theta[(3+\cos 2 \theta) \cos \varphi-(1-\cos 2 \theta) \cos 3 \varphi] ; \\
& \Psi_{36}=-\frac{3}{16} \sin 2 \theta[(3+\cos 2 \theta) \sin \varphi-(1-\cos 2 \theta) \sin 3 \varphi] \\
& \Psi_{44}=\frac{3}{4} \sin ^{2} \theta\left[\left(1+2 \cos ^{2} \theta\right)+\left(1-2 \cos ^{2} \theta\right) \cos 2 \varphi\right] \\
& \Psi_{45}=\frac{3}{16} \sin 2 \theta[(3+\cos 2 \theta) \sin \varphi+(1-\cos 2 \theta) \sin 3 \varphi] ; \\
& \Psi_{46}=\frac{3}{16} \sin 2 \theta[(3+\cos 2 \theta) \cos \varphi+(1-\cos 2 \theta) \cos 3 \varphi] \\
& \Psi_{55}=\frac{3}{16}\left[\left(1+6 \cos ^{2} \theta+\sin ^{4} \theta+\cos ^{4} \theta\right)+\left(1-2 \cos ^{2} \theta+\cos ^{4} \theta+\sin ^{4} \theta\right) \cos 4 \varphi\right] ; \\
& \Psi_{56}=-\frac{3}{16}\left[\left(1-2 \cos ^{2} \theta+\cos ^{4} \theta+\sin ^{4} \theta\right) \sin 4 \varphi\right] \\
& \Psi_{66}=\frac{3}{16}\left[\left(1+6 \cos ^{2} \theta+\sin ^{4} \theta+\cos ^{4} \theta\right)-\left(1-2 \cos ^{2} \theta+\cos ^{4} \theta+\sin ^{4} \theta\right) \cos 4 \varphi\right] .
\end{aligned}
$$

The elements of the matrix $\hat{\Psi}$ satisfy certain symmetry properties with respect to the main diagonal. Hence the number of independent elements are only 21. 


\section{REFERENCES}

Anusha, L. S., \& Nagendra, K. N. 2011, ApJ, 726, 6, Paper I

Anusha, L. S., Nagendra, K. N., \& Paletou, F. ApJ, 2011, 726, 96, Paper II

Auer, L. H., \& Paletou, F. 1994, A\&A, 285, 675

Bommier, V. 1997, A\&A, 328, 706

Bommier, V. 1997b, A\&A, 328, 726

Chandrasekhar, S. 1960, Radiative Transfer (New York: Dover)

Dittmann, O. J. 1999, in Solar Polarization, ed. K. N. Nagendra \& J. O. Stenflo (Boston: Kluwer) , 201

Domke, H., \& Hubeny, I. 1988, ApJ, 334, 527

Faurobert, M. 1988, A\&A, 194, 268

Faurobert-Scholl, M. 1991, A\&A, 246, 469

Faurobert-Scholl, M. 1992, A\&A, 258, 521

Fluri, D. M., Nagendra, K. N., \& Frisch, H. 2003, A\&A, 400, 303

Frisch, H. 2007, A\&A, 476, 665 (HF07)

Hummer, D. G. 1962, MNRAS, 125, 21

Landi Degl'Innocenti, E., \& Landolfi, M. 2004, Polarization in Spectral Lines (Dordrecht: Kluwer)

Manso Sainz, R., \& Trujillo Bueno, J. 1999, in Solar Polarization, ed. K. N. Nagendra \& J. O. Stenflo (Boston: Kluwer), 143

Nagendra, K. N., Frisch, H., \& Faurobert-Scholl, M. 1998, A\&A, 332, 610

Nagendra, K. N., Paletou, F., Frisch, H., \& Faurobert-Scholl, M. 1999, in Solar Polarization, ed. K. N. Nagendra \& J. O. Stenflo (Boston: Kluwer), 127

Nagendra, K. N., Frisch, H., \& Faurobert, M. 2002, A\&A, 395, 305

Nagendra, K. N., Frisch, H., \& Fluri, D. M. 2003, in ASP Conf. Ser. 307, Solar Polarization 3, ed. J. Trujillo Bueno \& J. Sánchez Almeida (San Francisco: ASP), 227
Stenflo, J. O. 1994, Solar Magnetic Fields: Polarized Radiation Diagnostics (Dordrecht: Kluwer)

This 2-column preprint was prepared with the AAS LATEX macros v5.2. 

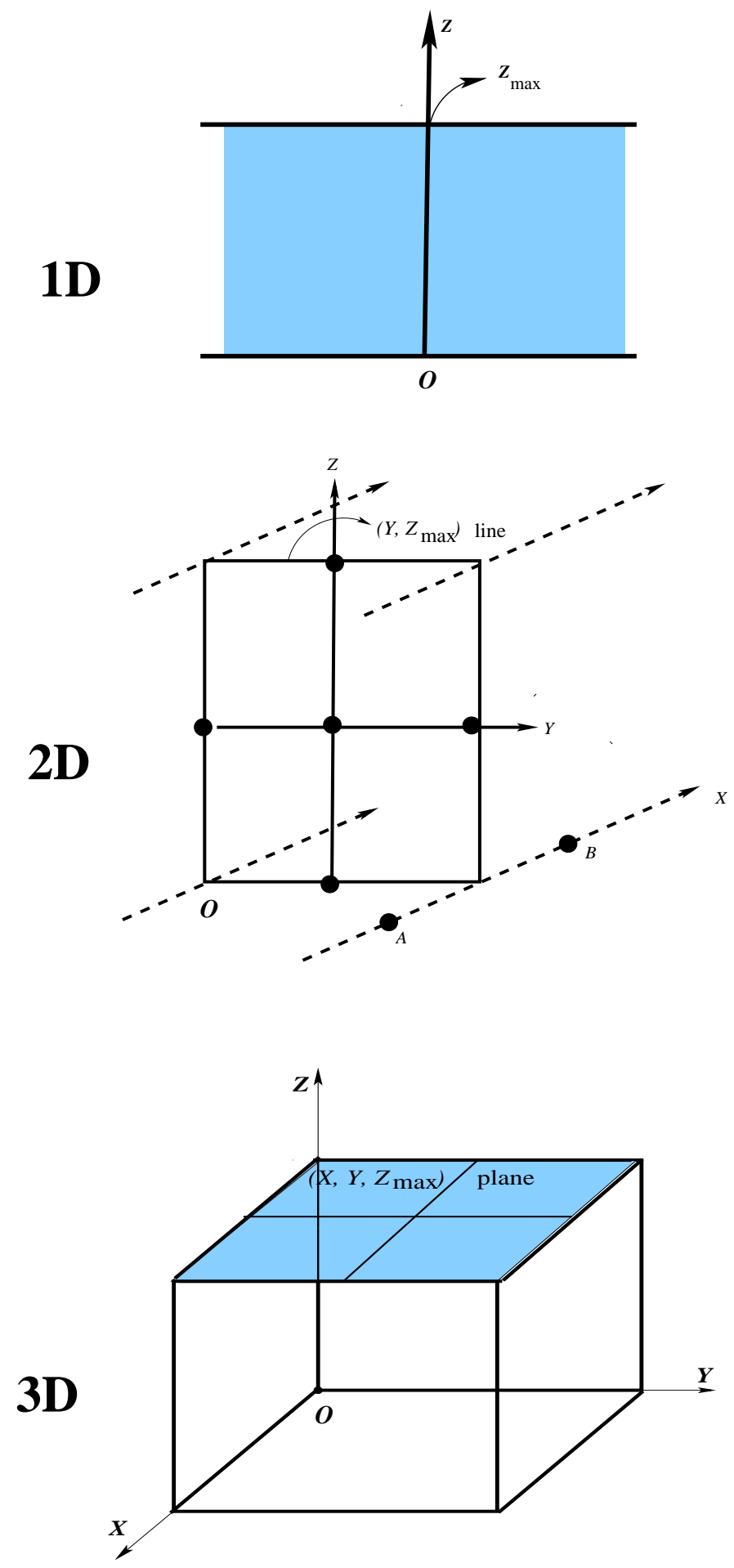

Fig. 1.- The $\mathrm{RT}$ in $1 \mathrm{D}, 2 \mathrm{D}$ and $3 \mathrm{D}$ geometries. The $Z_{\max },\left(Y, Z_{\max }\right)$, and $\left(X, Y, Z_{\max }\right)$ represent respectively, the point, the line, and the plane on which the emergent solutions are shown in this paper. The corresponding atmospheric reference frame is shown in Figure 2. The points A and B marked on the 2D geometry figure represent an example of the spatial points where the symmetry of the polarized radiation field (Equation 26) is valid in a $2 \mathrm{D}$ medium. 
Table 1: The 12-point Carlsson type B quadrature for the azimuth angle $\varphi$. The corresponding values of $\sin \varphi, \cos \varphi, \sin 2 \varphi$ and $\cos 2 \varphi$ are given for the purpose of discussion.

\begin{tabular}{crrrr}
\hline \hline$\varphi_{i}$ (in degrees) & $\sin \varphi$ & $\cos \varphi$ & $\sin 2 \varphi$ & $\cos 2 \varphi$ \\
\hline \hline 30 & 0.5 & 0.866 & 0.866 & 0.5 \\
\hline 45 & 0.707 & 0.707 & 1 & 0 \\
\hline 60 & 0.866 & 0.5 & 0.866 & -0.5 \\
\hline 120 & 0.866 & -0.5 & -0.866 & -0.5 \\
\hline 135 & 0.707 & -0.707 & -1 & 0 \\
\hline 150 & 0.5 & -0.866 & -0.866 & 0.5 \\
\hline 210 & -0.5 & -0.866 & 0.866 & 0.5 \\
\hline 225 & -0.707 & -0.707 & 1 & 0 \\
\hline 240 & -0.866 & -0.5 & 0.866 & -0.5 \\
\hline 300 & -0.866 & 0.5 & -0.866 & -0.5 \\
\hline 315 & -0.707 & 0.707 & -1 & 0 \\
\hline 330 & -0.5 & 0.866 & -0.866 & 0.5 \\
\hline
\end{tabular}

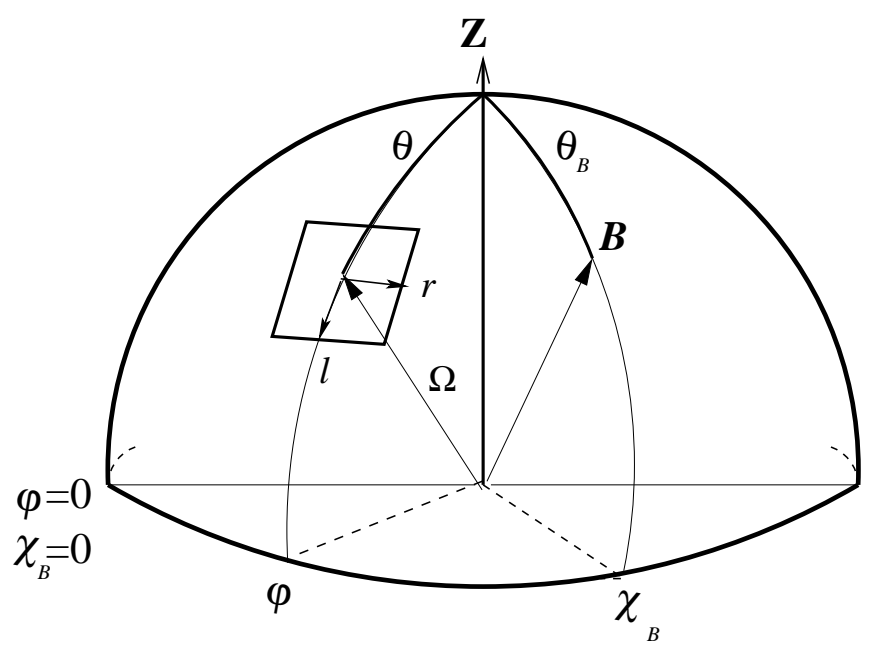

Fig. 2.- The atmospheric reference frame. The angle pair $(\theta, \varphi)$ define the outgoing ray direction. The magnetic field is characterized by $\boldsymbol{B}=\left(\Gamma, \theta_{B}, \chi_{B}\right)$, where $\Gamma$ is the Hanle efficiency parameter and $\left(\theta_{B}, \chi_{B}\right)$ defines the field direction. $\Theta$ is the scattering angle. 


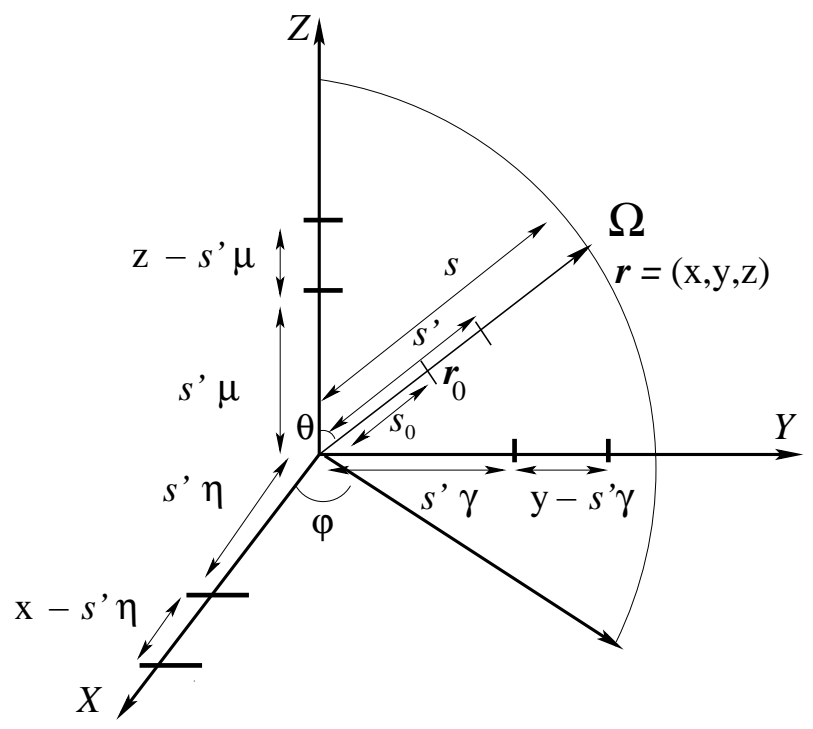

Fig. 3. - The definition of the position vector $\boldsymbol{r}$ and the projected distances $\boldsymbol{r}-s^{\prime} \boldsymbol{\Omega}$ which appear in Equation 6. $\boldsymbol{r}_{0}$ and $\boldsymbol{r}$ are the arbitrary initial and final locations that appear in formal solution integral (Equation 6).

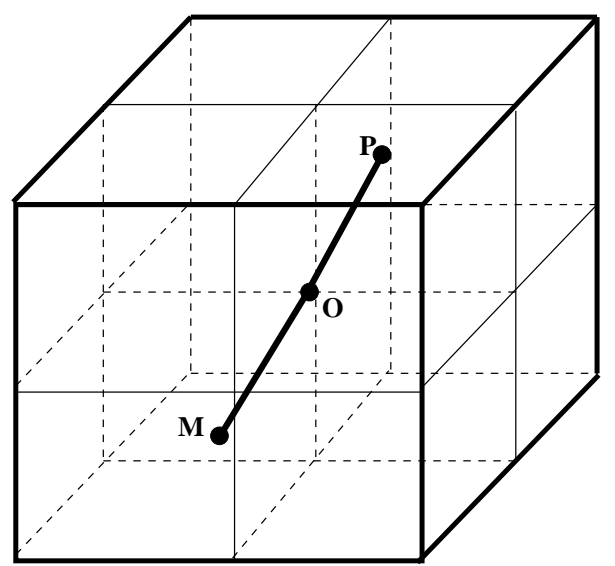

Fig. 4.- An elemental cube, showing the transfer along a section of the ray path, called a short characteristic (MOP). The quantities $\mathcal{S}, \kappa_{\text {tot }}$ at $\mathrm{M}$ and $\mathrm{P}$, and $\mathcal{I}_{M}$ at $\mathrm{M}$ are computed using parabolic interpolation formulae as $\mathrm{M}$ and $\mathrm{P}$ are non-grid points. 


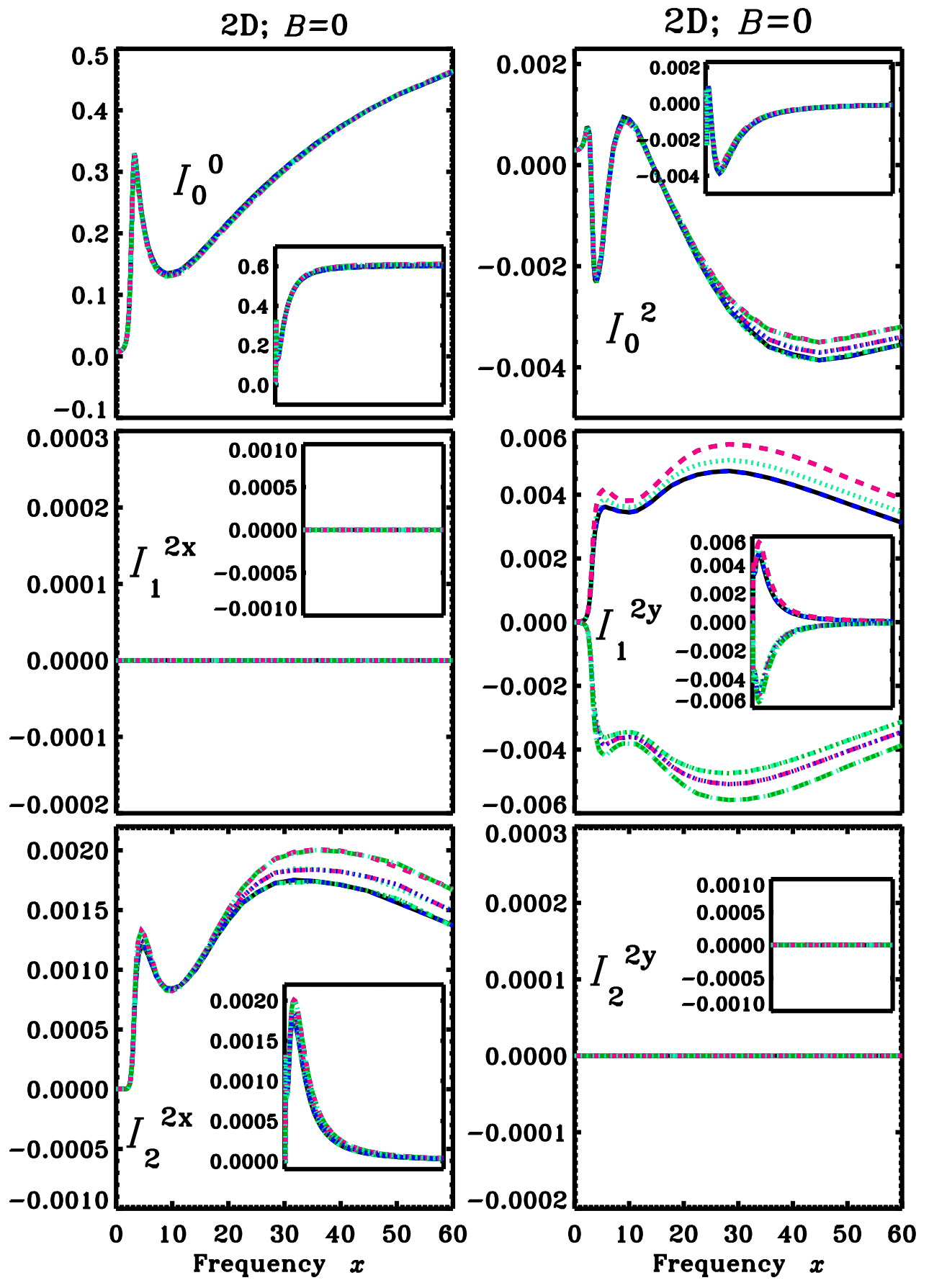

Fig. 5.- The emergent, spatially averaged irreducible Stokes vector components formed in a non-magnetic 2D medium. Different curves represent different values of the radiation azimuth $\varphi$. The value of $\mu=0.11$. The other model parameters are given in Section 6.1. The inset panels show the far wing behavior of $\mathcal{I}$. The $x$ grid for these inset panels is $0 \leq x \leq 600$. 

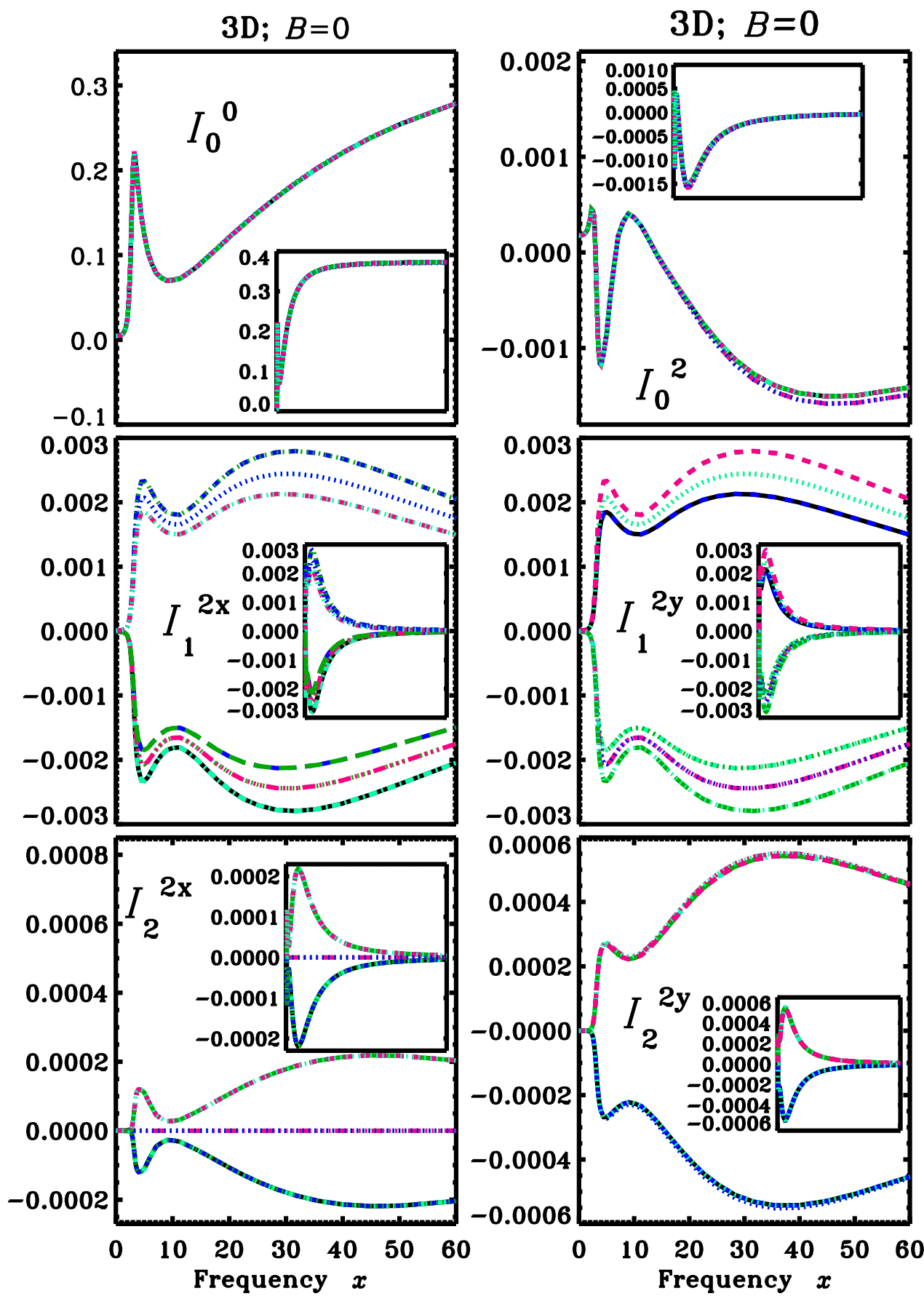

Fig. 6. - Same as Figure 5 but for a 3D medium. 
(a) $1 \mathrm{D} ; B=0$

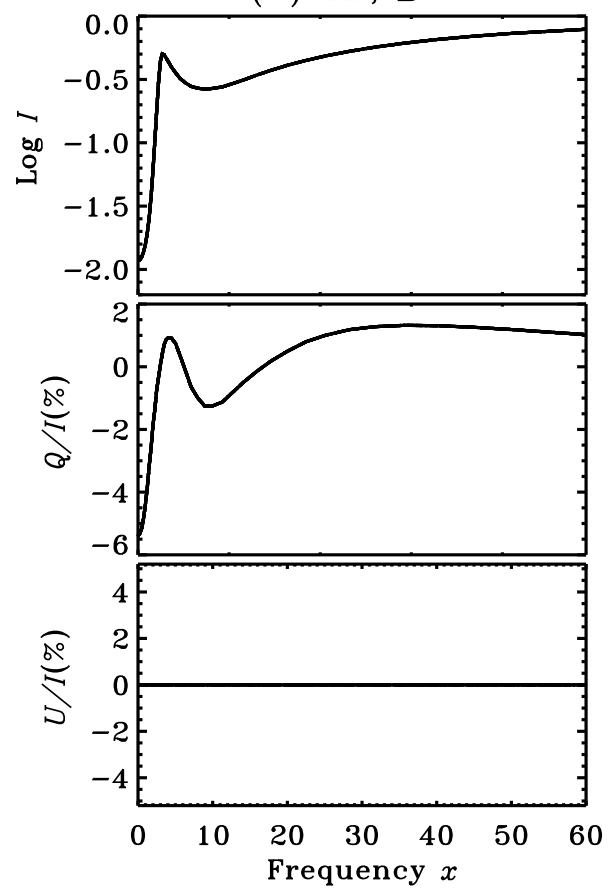

(b) $2 \mathrm{D} ; B=0$

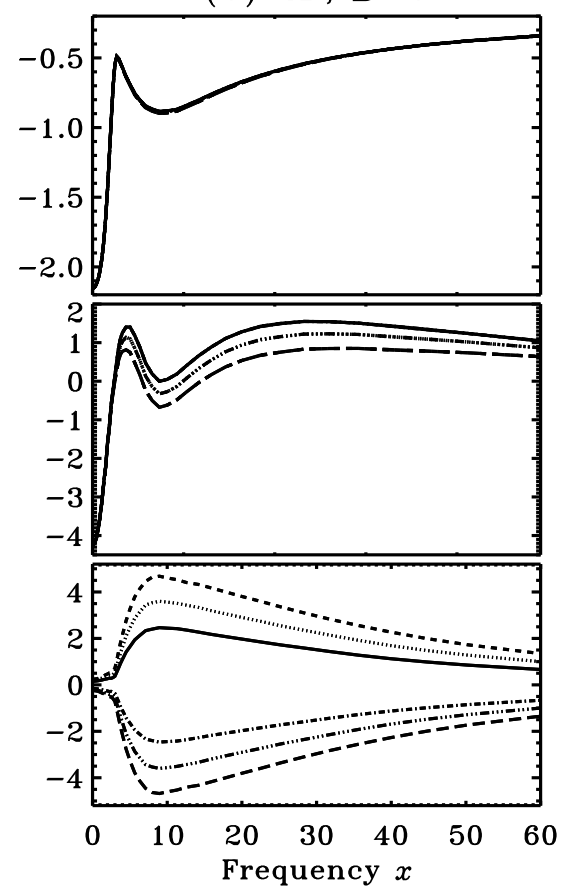

(c) $3 \mathrm{D} ; B=0$

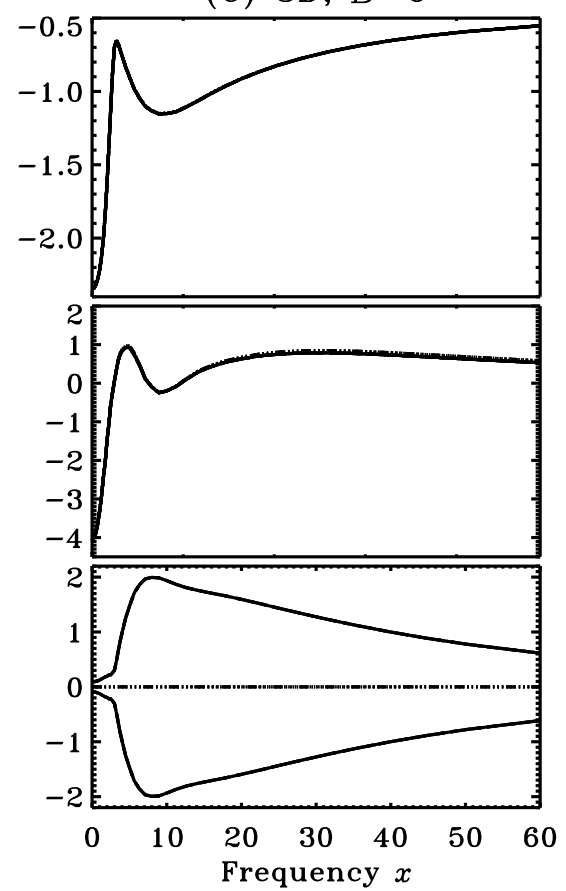

Fig. 7.- The emergent, spatially averaged $(I, Q / I, U / I)$ in non-magnetic $1 \mathrm{D}, 2 \mathrm{D}$ and $3 \mathrm{D}$ media. Different curves represent different values of the radiation azimuth $\varphi$. The value of $\mu=0.11$. The other model parameters are given in Section 6.1. 

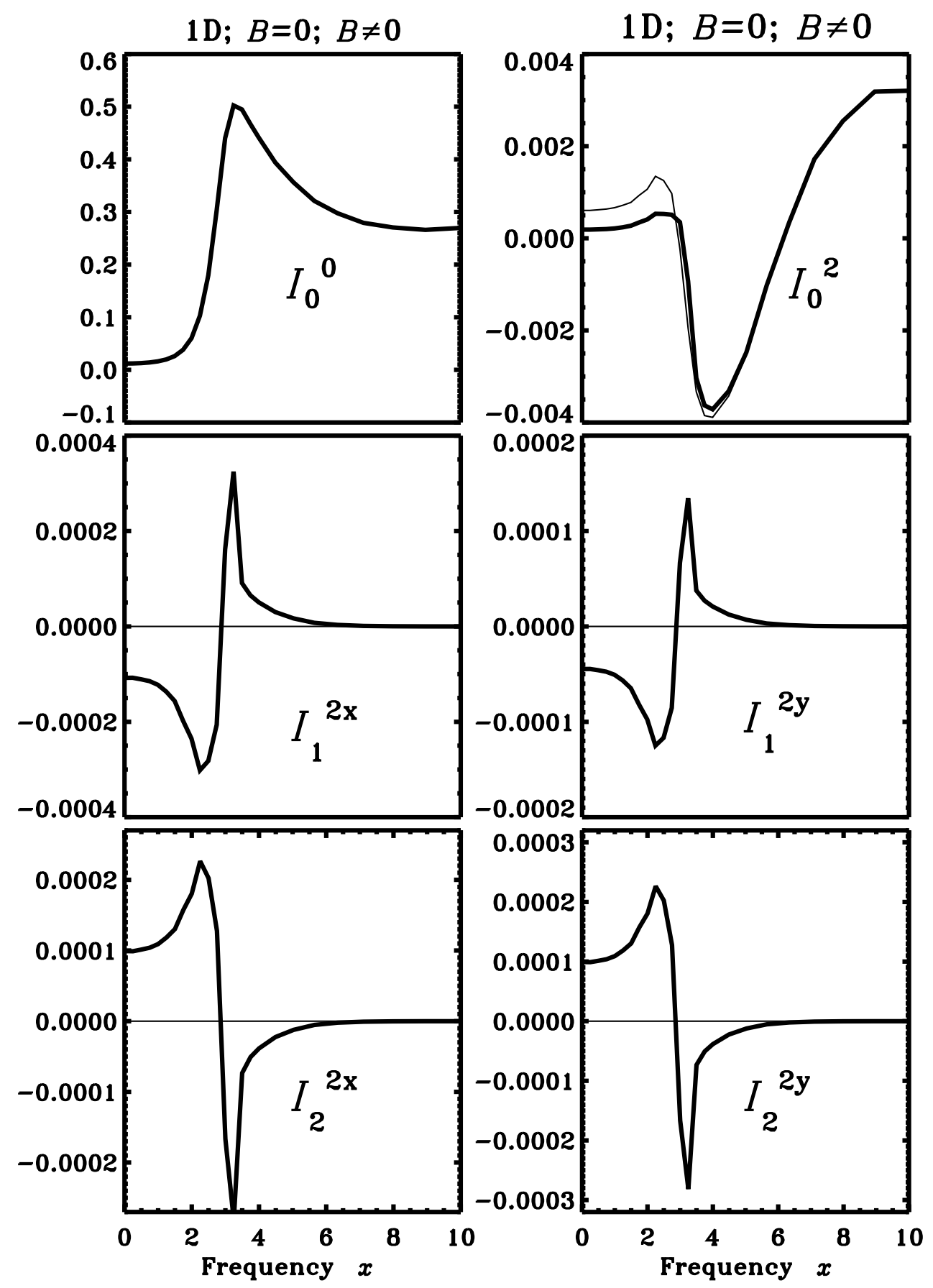

Fig. 8. - Same as Figure 5 but for a magnetic 1D medium. The vector magnetic field is represented by $\left(\Gamma, \theta_{B}, \chi_{B}\right)=\left(1,90^{\circ}, 68^{\circ}\right)$. The thin solid lines show the corresponding non-magnetic components. 


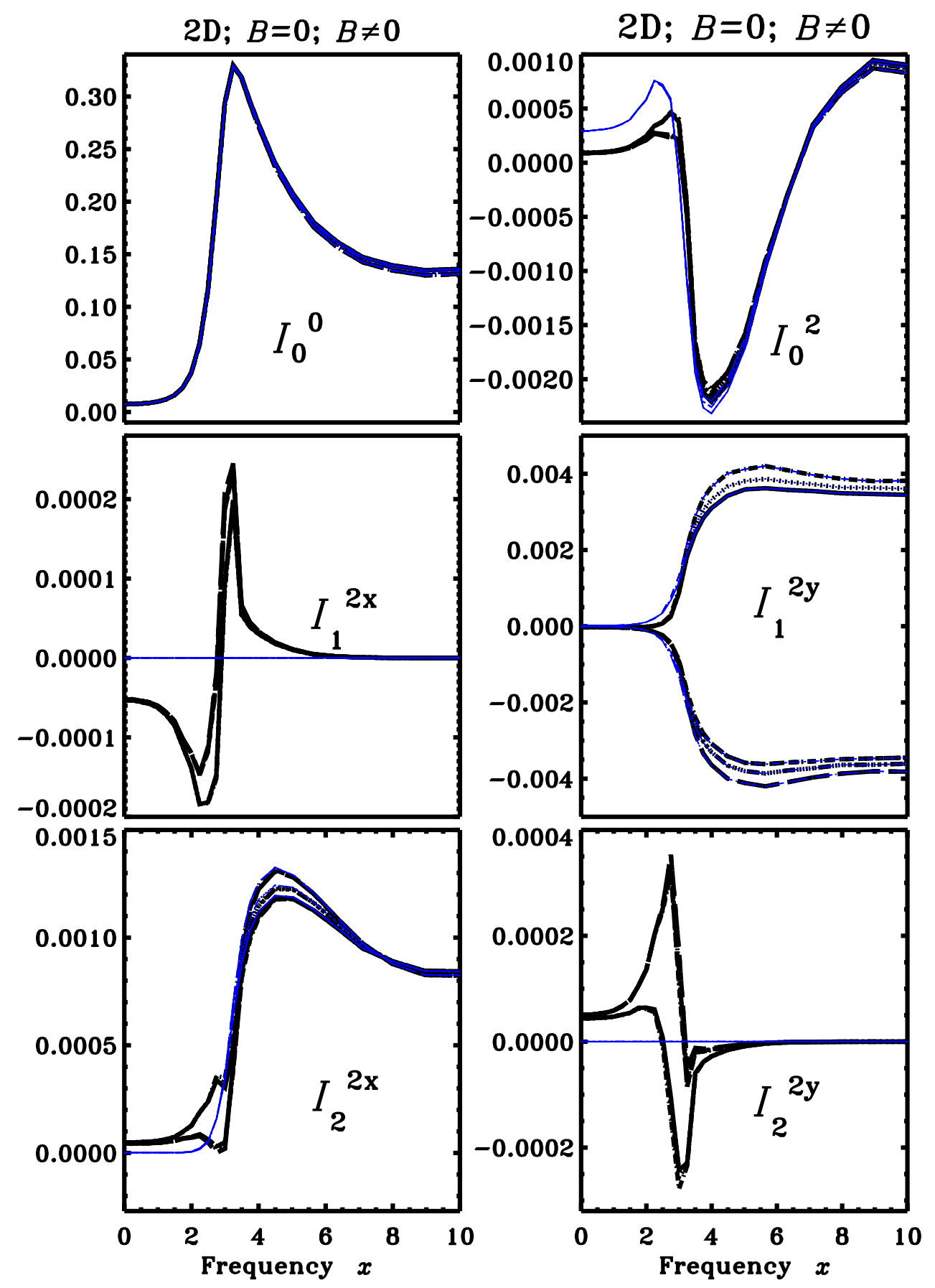

Fig. 9.- Same as Figure 8 but for a 2D medium. 


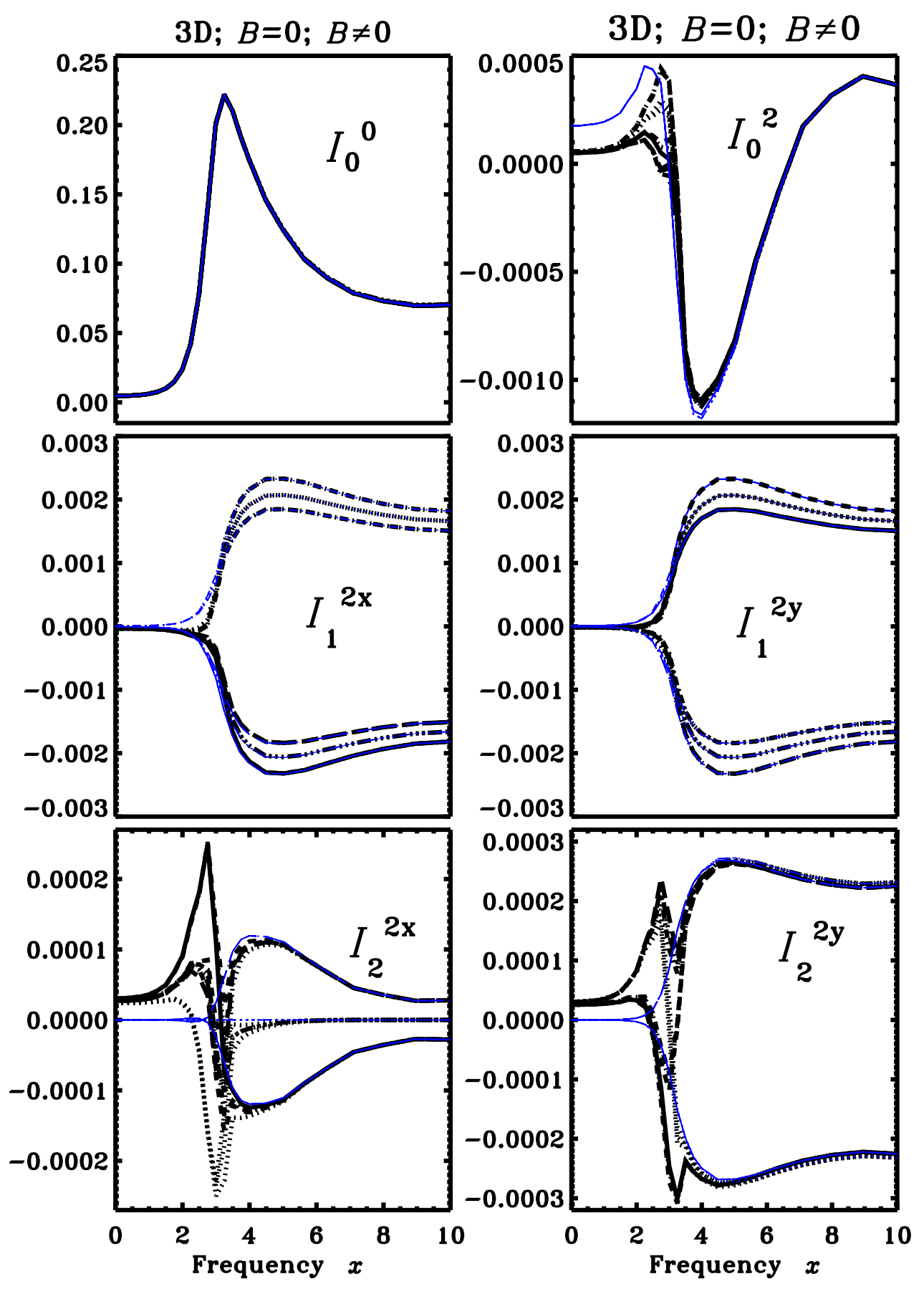

Fig. 10.- Same as Figure 8 but for a 3D medium. 

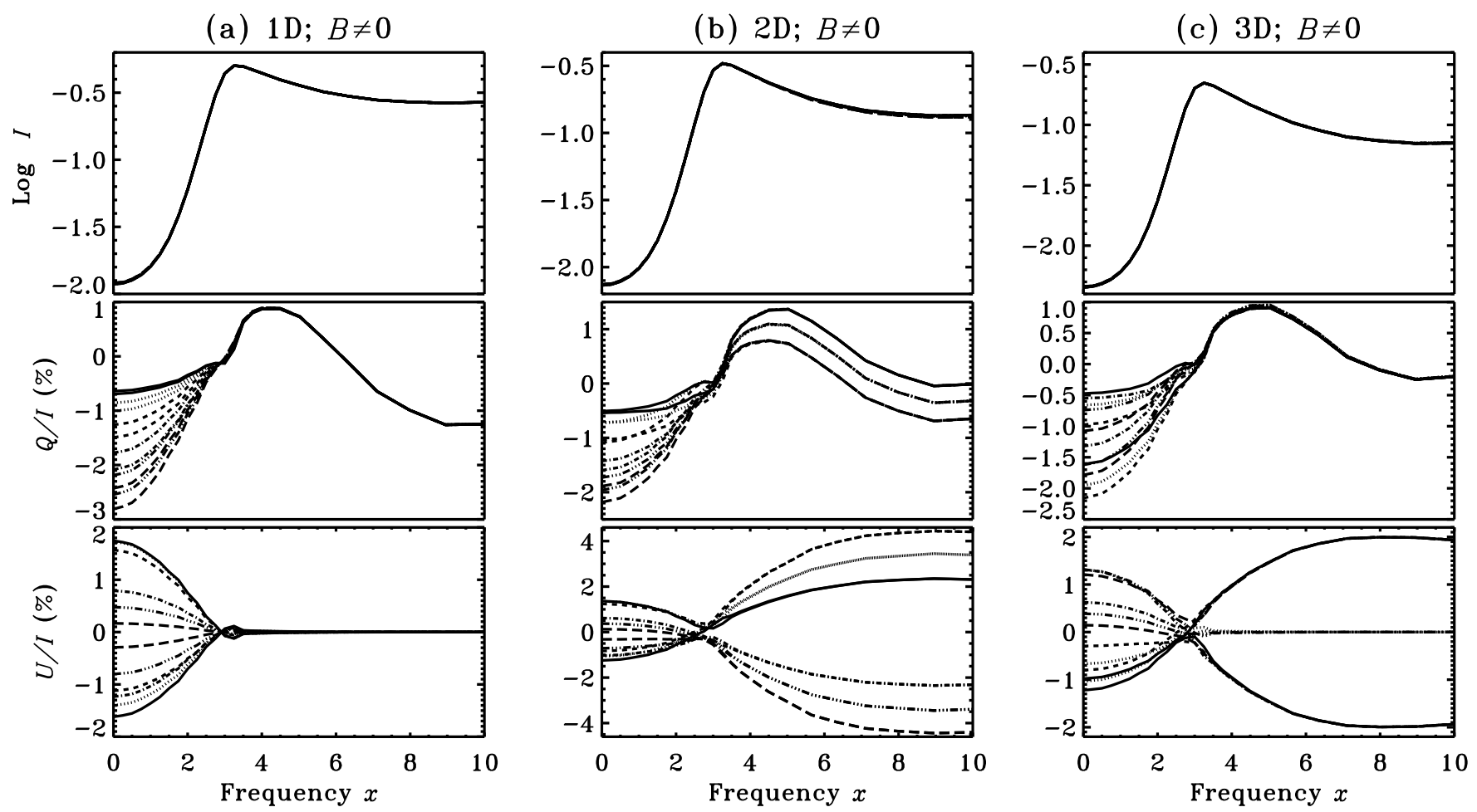

Fig. 11.- A comparison of emergent $I, Q / I$ and $U / I$ profiles formed in a magnetized $1 \mathrm{D}$ media with the emergent, spatially averaged $I, Q / I$ and $U / I$ formed in a magnetized $2 \mathrm{D}$ and $3 \mathrm{D}$ media. The model parameters are same as in Figure 8. 

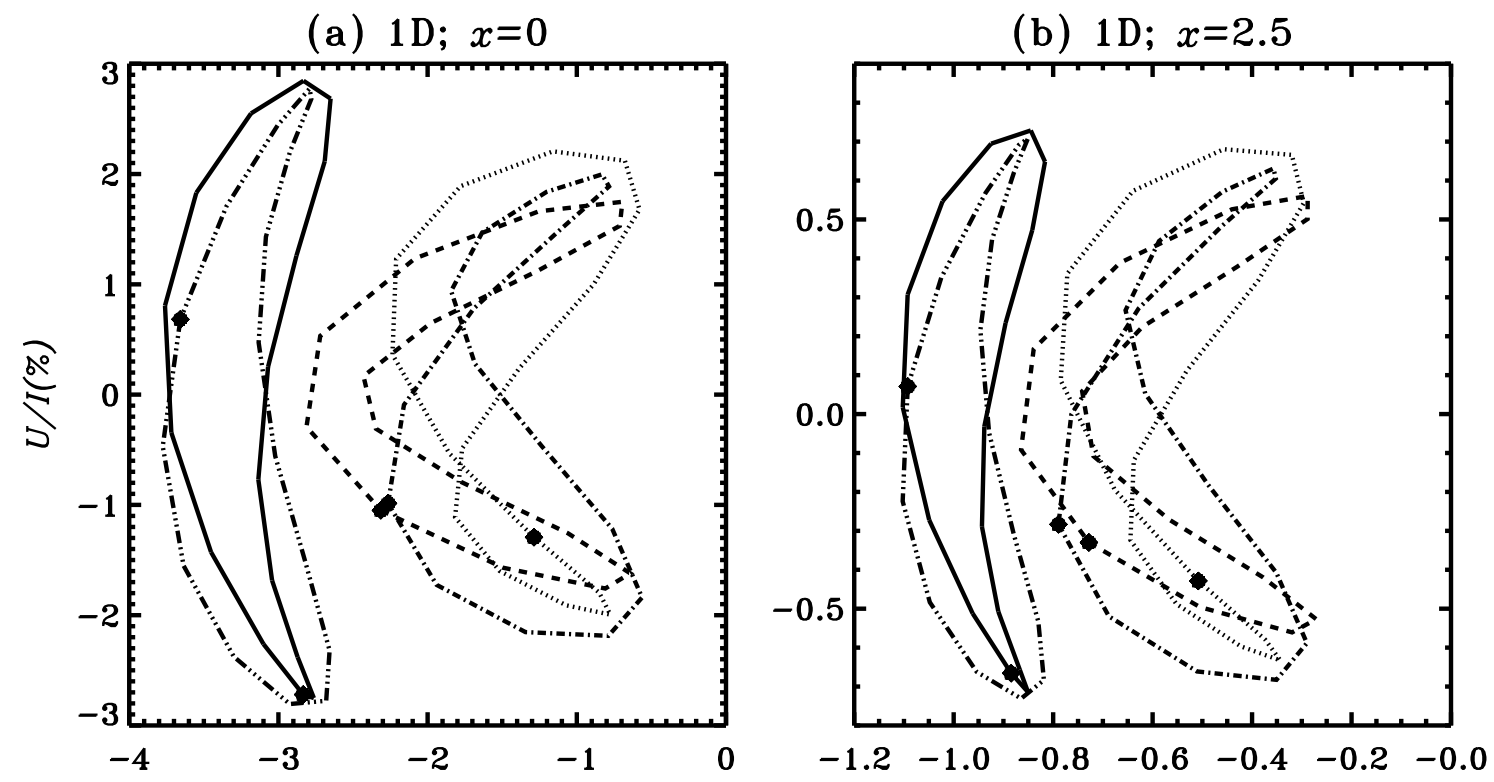

(c) $2 \mathrm{D} ; x=0$

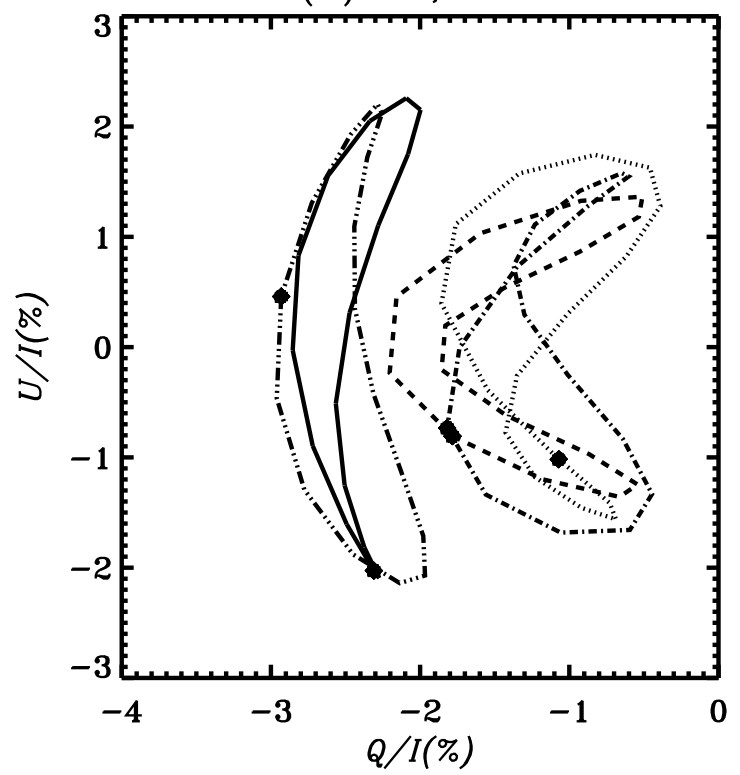

(d) $2 \mathrm{D} ; x=2.5$

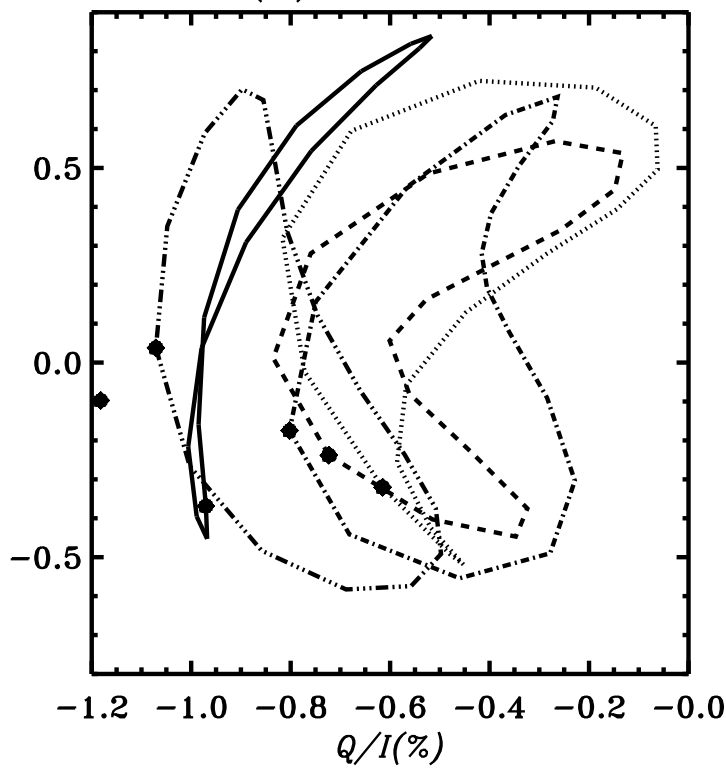

Fig. 12.- A comparison of the polarization diagrams in 1D and 2D media for two different values of frequency $x$. In $2 \mathrm{D}$, the spatially averaged quantities are shown. The magnetic field parameters are given by $\Gamma=1$, five values of $\theta_{B}$ in the range $30^{\circ}$ to $150^{\circ}$ in steps of $30^{\circ}$, seventeen values of $\chi_{B}$ in the range $0^{\circ}$ to $360^{\circ}$ in steps of $22^{\circ} .5$. Different line types correspond to different values of $\theta_{B}$. Heavy square symbol represents $\chi_{B}=0$, and as we move in the counter-clockwise direction, $\chi_{B}$ takes increasingly larger values. The ray direction is specified by $(\mu, \varphi)=\left(0.11,60^{\circ}\right)$. The line types represent different $\theta_{B}$, namely (solid, dotted, dashed, dot-dashed, dash-triple-dotted $)=\left(30^{\circ}, 60^{\circ}, 90^{\circ}, 120^{\circ}, 150^{\circ}\right)$. 

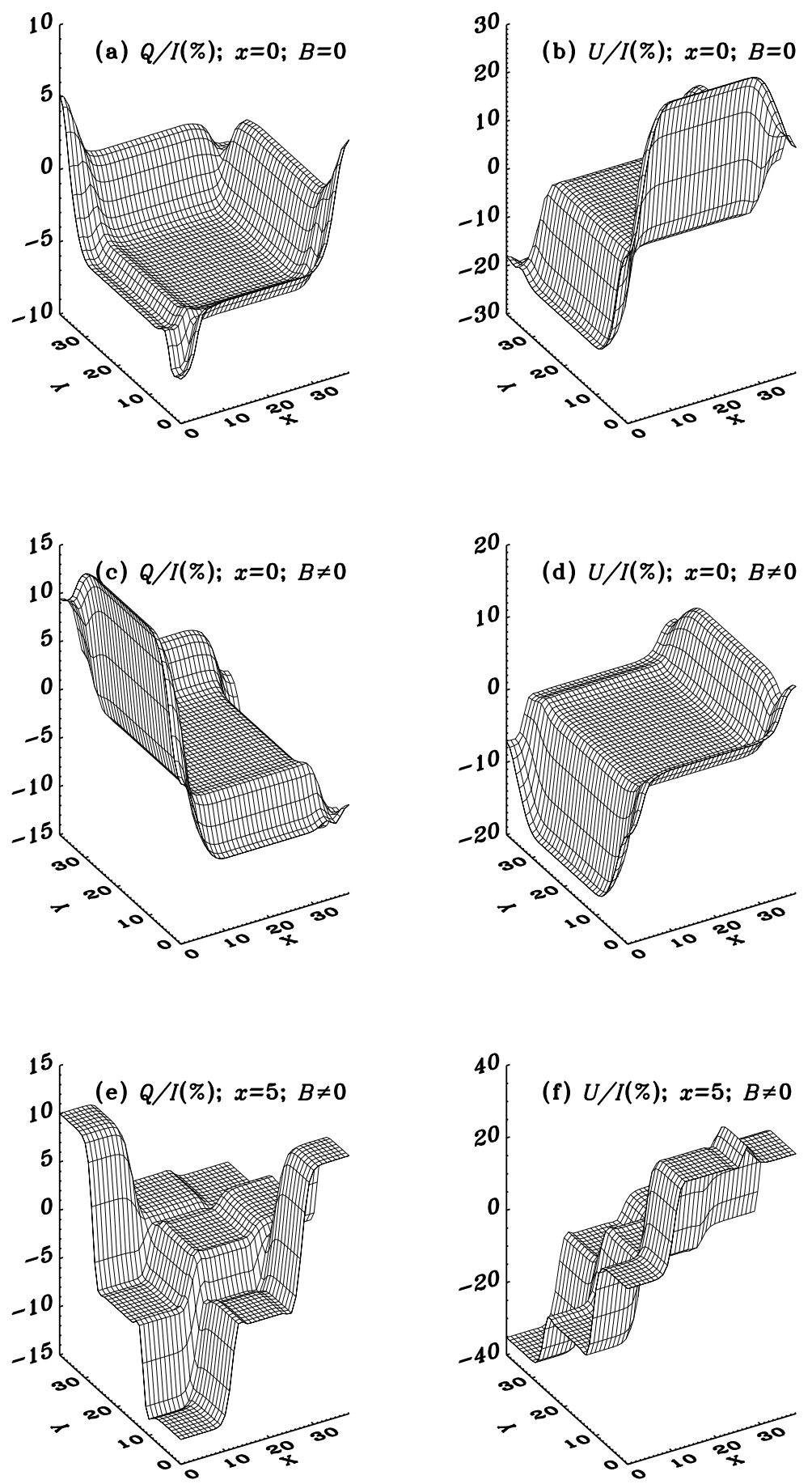

Fig. 13.- The spatial distribution of $(Q / I, U / I)$ on the top surface of a $3 \mathrm{D}$ medium. The $Q / I$ and $U / I$ are plotted as a function of the grid indices of $\tau_{X}$ and $\tau_{Y}$. The ray direction is specified by $(\mu, \varphi)=\left(0.11,60^{\circ}\right)$. Panels (a) and (b) demonstrate purely the multi-D effects. Panels (c) and (d) demonstrate the magnetic field effects. Panels (e) and (f) demonstrate the PRD effects. See Section 6.3 for details. 

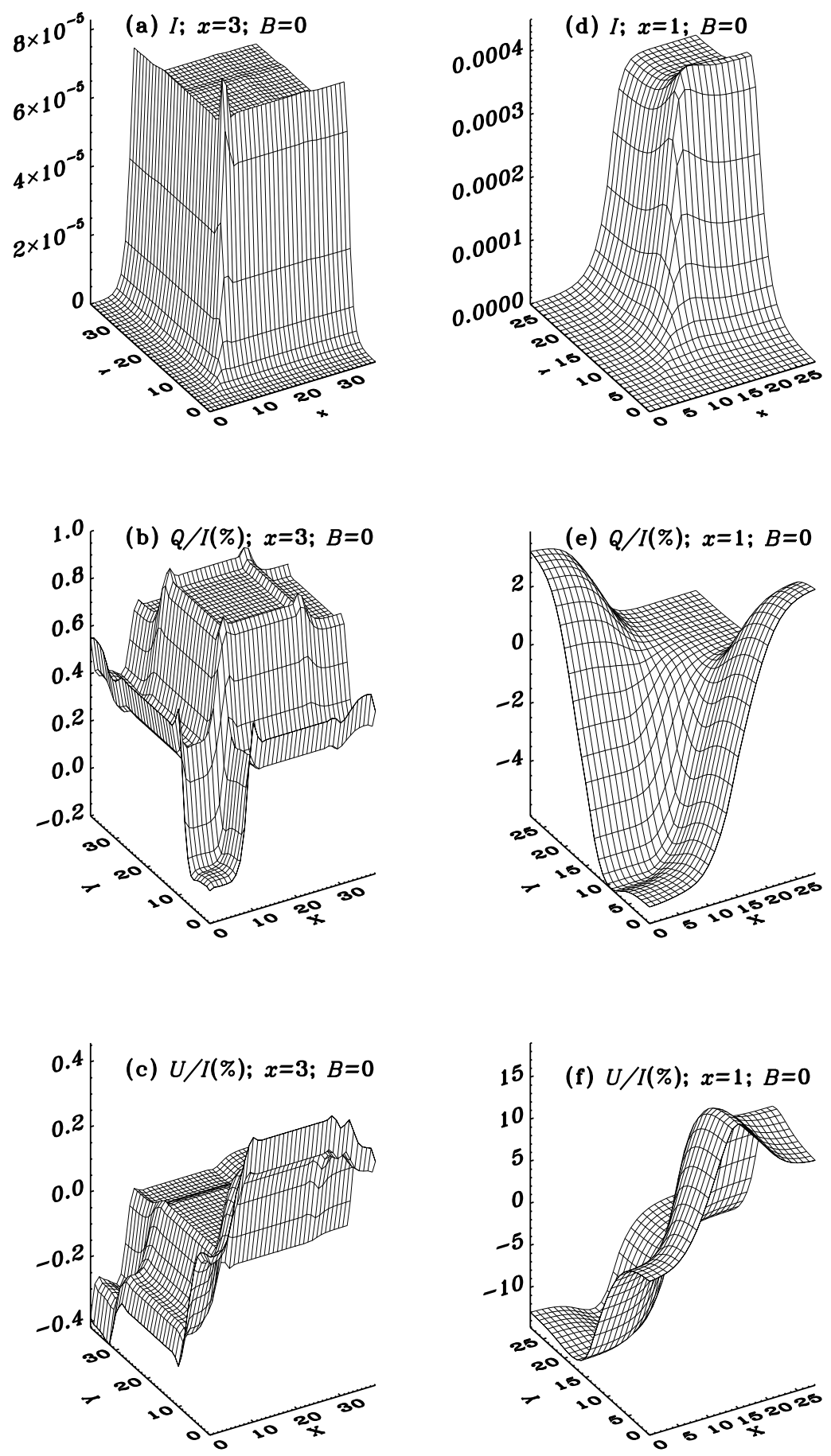

Fig. 14. - The spatial distribution of $(Q / I, U / I)$ on the top surface of a 3D media. The $Q / I$ and $U / I$ are plotted as a function of the grid indices of $\tau_{X}$ and $\tau_{Y}$. The ray (viewing) direction is specified by $(\mu, \varphi)=\left(0.11,60^{\circ}\right)$. Left panels represent a sheet structure and right panels represent a rod structure when viewed along the $\pm Z$ direction. 